\title{
Equilibrium States of Gravitational Systems
}

\author{
Heide Narnhofer ${ }^{1}$ and Geoffrey L. Sewell ${ }^{2}$ \\ 1 Institut für Theoretische Physik, Universität Wien, Wien, Austria \\ 2 Department of Physics, Queen Mary College, London, England
}

\begin{abstract}
We formulate the equilibrium correlation functions for local observables of an assembly of non-relativistic, neutral gravitating fermions in the limit where the number of particles becomes infinite, and in a scaling where the region $\Omega$, to which they are confined, remains fixed. We show that these correlation functions correspond, in the limit concerned, to states on the discrete tensor product $\bigotimes_{x \in \Omega} \mathscr{A}_{x}$, where the $\mathscr{A}_{x}^{\prime}$ s are copies of the gauge invariant $C^{*}$-algebra $\mathscr{A}$ of the CAR over $L^{2}\left(\mathbf{R}^{3}\right)$. The equilibrium states themselves are then given by $\bigotimes_{x \in \Omega} \bar{\omega}_{\varrho_{0}(x)}$, where $\bar{\omega}_{\varrho}$ is the Gibbs state on $\mathscr{A}$ for an infinitely extended ideal Fermi gas at density $\varrho$, and where $\varrho_{0}$ is the normalised density function that minimises the Thomas-Fermi functional, obtained in [2], governing the equilibrium thermodynamics of the system.
\end{abstract}

\section{Introduction}

The thermodynamical limiting behaviour of a non-relativistic assembly of $N$ neutral, gravitating fermions of one species, confined to a suitably regular bounded three-dimensional domain $\Omega$, is not of the usual type, since the internal energy, temperature and volume of the system scale like $N^{7 / 3}, N^{4 / 3}$ and $N^{-1}$, respectively, as $N \rightarrow \infty$ [1-4]. The system also possesses simple properties of scale invariance. In the particular scaling where the domain $\Omega$ and the temperature are fixed, while the particle mass and gravitational constant become proportional to $N^{2 / 3}$ and $N^{-1}$, respectively, the specific free energy tends, as $N \rightarrow \infty$, to the minimum value of the Thomas-Fermi functional $\Phi_{0}$ on the bounded probability densities on $\Omega$, given by the formula

$$
\Phi_{0}(\varrho)=\int_{\Omega} d^{3} x \varphi_{0}(\varrho(x))-\frac{1}{2} \int_{\Omega^{2}} d^{3} x d^{3} y \frac{\varrho(x) \varrho(y)}{|x-y|},
$$

where $\varphi_{0}(\varrho)$ is the equilibrium free energy density of an ideal Fermi gas at density $\varrho$ and at the given temperature, $T$. According to a numerical solution of the resultant 
Euler equation [3] for the case where $\Omega$ is spherical, the system undergoes a phase transition at a temperature $T_{c}$; and for $T \neq T_{c}$, the probability density function that minimises $\Phi_{0}$ is unique. Furthermore, it has been proved [5] that, whenever $\Phi_{0}$ is minimised at a unique probability density $\varrho_{0}$, then this latter function corresponds to the normalised equilibrium density distribution of the system in the limit $N \rightarrow \infty$; while the normalised densities at different points of $\Omega$ become uncorrelated in this limit.

The purpose of the present paper is to formulate the equilibrium states of the system, in the limit $N \rightarrow \infty$, in the same scaling, described above, that was used in $[4,5]$. Here, a state means a positive normalised linear functional on the $C^{*}$-algebra of observables of the system, but in view of the chosen scaling, this algebra is not taken to be that of the CAR over $L^{2}(\Omega)$ : for as the system consists of an infinity of particles confined to a bounded region, its states could not possibly be locally normal ones on the latter algebra [6]. In fact, we arrive at our specifications of both the algebra of observables and the equilibrium states of the infinite assembly of particles in through a treatment of the limiting form, as $N \rightarrow \infty$, of the equilibrium correlation functions of localised observables of the finite system, that are transformed to a scaling where the length unit is the mean interparticle spacing (cf. Sect. 2). In this way we arrive at the conclusion that the algebra of observables of the infinite system is given by the discrete tensor product $\bigotimes_{x \in \Omega} \mathscr{A}_{x}$, where the $\mathscr{A}_{x}$ 's are copies of the gauge-invariant $C^{*}$-algebra, $\mathscr{A}$, of the CAR over $L^{2}\left(\mathbf{R}^{3}\right)$; and that, if the Thomas-Fermi functional $\Phi_{0}$ for the given temperature is minimised at the unique probability density $\varrho_{0}$, then the equilibrium state of the system is $\bigotimes_{x \in \Omega} \bar{\omega}_{\varrho_{0}}(x)$, where $\bar{\omega}_{\varrho}$ is the Gibbs state on corresponding to particle number density $\varrho$.

The essential reason why the rescaled observables correspond to $\bigotimes_{x \in \Omega} \mathscr{A}_{x}$ may be understood as follows. In the limit $N \rightarrow \infty$, every neighbourhood of a point $x(\in \Omega)$ contains an infinity of particles. Thus, when the observables are suitably rescaled, it transpires that, in this limit, each point $x(\in \Omega)$ carriers with it an algebra of observables $\mathscr{A}_{x}$, given by a copy of $\mathscr{A}$, while the algebra of observables for the entire system is $\bigotimes_{x \in \Omega} \mathscr{A}_{x}$. The elements $x$ of $\Omega$, as they appear in this discrete tensor product, should be regarded as points in the hydrodynamical sense, since the algebra of observables attached to each of them corresponds to that of an infinite system. Accordingly, we term this tensor product the hydro-local algebra of observables, and denote it by $\mathscr{H} \mathscr{L}(\mathscr{A})$. Further, the equilibrium state $\bigotimes_{x \in \Omega} \bar{\omega}_{\varrho_{0}}(x)$ that we obtain is characterised by the properties that, at each point $x(\in \Omega)$ it reduces to that of an ideal Fermi gas at the prevailing local density $\varrho_{0}(x)$; and that it carries no correlations between the observables attached to different points of $\Omega$.

The subject-matter of the article will be organised as follows. In Sect. 2, we shall formulate the model and state the main theorem, yielding the limiting form as $N \rightarrow \infty$ of the equilibrium correlation functions for the re-scaled local observables and the resultant state $\bar{\omega}:=\bigotimes_{x \in \Omega} \bar{\omega}_{\varrho_{0}}(x)$ on $\mathscr{H} \mathscr{L}(\mathscr{A})$. We shall then discuss this theorem and argue that $\bar{\omega}$ is an equilibrium state, not only because it represents a 
limiting form of Gibbs states, but also by virtue of its various stability properties : we shall also make a conjecture concerning the possibility that the system supports states that are metastable in the sense of being locally but not globally stable (cf. [7]). In Sect. 3, we shall re-cast the theorem of Sect. 2 as consequences of other theorems concerned with the linear response of the gravitational system to certain perturbations. In Sect. 4 , we shall make a number of constructions, leading to further auxilliary theorems and lemmas. In Sects. 5 and 6, we shall present the proofs of the theorems and lemmas, respectively, of the two previous Sections. The two Appendices are devoted to self-contained treatments of non-gravitational systems, that yield results required for the proofs of Sects. 4 and 5. Thus, in Appendix 1, we shall employ a generalisation of the methods of [8] to establish that the properties of a certain class of models are given by a mean field theory; and, in Appendix 2, we shall introduce a construction, analogous to that used in [9] for the treatment of equilibrium states of lattice systems, to prove the uniqueness of the translationally invariant equilibrium state of an ideal Fermi gas.

Finally, we remark that the whole theory presented here may easily be generalised, as in [4], to two-component systems of charged gravitational particles, for which the total charge is zero.

\section{The Model}

Let $\mathscr{G}_{N}$ be an assembly of $N$ non-relativistic gravitational fermions of one species, enclosed in a bounded, connected, three-dimensional region $\Omega$. In the scaling where $\Omega$ is fixed and the particle mass and gravitational constant are proportional to $N^{2 / 3}$ and $N^{-1}$, respectively, the Hamiltonian for $\mathscr{G}_{N}$ is the operator in the Hilbert space $\mathscr{H}_{N}(\Omega)$ of antisymmetric square-integrable functions on $\Omega^{N}$, given by the formula (cf. $[4,5]$ )

$$
H_{N}=-\frac{1}{2} N^{-2 / 3} \sum_{j=1}^{N} \Delta_{j}+\frac{1}{2} N^{-1} \sum_{\substack{i, j=1 \\ i \neq j}}^{N} v\left(x_{i}, x_{j}\right),
$$

where

$$
v(x, y)=-|x-y|^{-1},
$$

and where Dirichlet boundary conditions are assumed. We define $\omega_{N}$ to be the Gibbs state on the bounded operators in $\mathscr{H}_{N}(\Omega)$, for temperature $\beta^{-1}$, i.e.

$$
\omega_{N}=\operatorname{Tr}\left((\cdot) e^{-\beta H_{N}}\right) / \operatorname{Tr}\left(e^{-\beta H_{N}}\right) .
$$

In order to relate the properties of $\mathscr{G}_{N}$, in the limit $N \rightarrow \infty$, to those of an ideal Fermi gas, $\mathscr{I}$, we introduce some definitions pertaining to the latter system. We take the algebra of observables, $\mathscr{A}$, for $\mathscr{I}$ to be the gauge-invariant $C^{*}$-algebra of the CAR over $L^{2}\left(\mathbf{R}^{3}\right)$. This algebra has a quasi-local structure $[10,11]$, i.e. it is the closure of the union, $\mathscr{A}_{L}$, of the $C^{*}$-algebras, $\mathscr{A}(\Lambda)$, of the CAR over the spaces $L^{2}(\Lambda)$, with $\Lambda\left(\subset \mathbf{R}^{3}\right)$ bounded and measurable. We identify $\mathscr{A}$ (resp. $\left.\mathscr{A}(\Lambda)\right)$ with its standard faithful representation in the Fock space $\mathscr{H}[$ resp. $\mathscr{H}(\Lambda) \subset \mathscr{H}]$ over $L^{2}\left(\mathbf{R}^{3}\right)$ [resp. $\left.L^{2}(\Lambda)\right]$. Here $\mathscr{A}(\Lambda), \mathscr{H}(\Lambda)$ are isotonic in $\Lambda$; and $\mathscr{H}$ [resp. $\mathscr{H}(\Lambda)]=\bigoplus_{0}^{\infty} \mathscr{H}_{n}\left[\right.$ resp. $\left.\bigoplus_{0}^{\infty} \mathscr{H}_{n}(\Lambda)\right]$, where $\mathscr{H}_{n}\left[\right.$ resp. $\left.\mathscr{H}_{n}(\Lambda)\right]$ is the Hilbert space of 
square-integrable antisymmetric functions on $\mathbf{R}^{3 n}$ (resp. $\Lambda^{n}$ ). We define the conditional expectation $\mathbf{E}(\cdot / \Lambda)$ to be the mapping from $\mathscr{A}$ onto $\mathscr{A}(\Lambda)$ given by

$$
(f, \mathbf{E}(A / \Lambda) g)=(f, A g) \forall A \in \mathscr{A} ; f, g \in \mathscr{H}(\Lambda) \text {. }
$$

For $\gamma \in \mathbf{R}_{+}$and $x \in \mathbf{R}^{3}$, we define $\sigma(\gamma, x)$ to be the automorphism of $\mathscr{A}$ implemented in $\mathscr{H}$ by the unitary operator $U(\gamma, x)$ according to the formulae

$$
\sigma(\gamma, x) A=U(\gamma, x) A U(\gamma, x)^{-1}
$$

where

$$
(U(\gamma, x) f)_{n}\left(x_{1}, \ldots, x_{n}\right)=\gamma^{3 n / 2} f_{n}\left(\gamma\left(x_{1}-x\right), \ldots, \gamma\left(x_{n}-x\right)\right)
$$

and $f_{n}$ is the $n$-particle component of $f$. For large $\gamma$, the automorphism $\sigma(\gamma, x)$ serves to concentrate the localisation of the observables around $x$ : in particular, for $x \in \operatorname{Int} \Omega$ and $A \in \mathscr{A}_{L}, \sigma(\gamma, x) A \in \mathscr{A}(\Omega)$ for $\gamma$ large enough.

Let $P_{N}$ be the projection operator from $\mathscr{H}(\Omega)$ onto $\mathscr{H}_{N}(\Omega)$, and let $\mathscr{A}_{N}(\Omega):=P_{N} \mathscr{A}(\Omega) P_{N}$. For $x \in \Omega$, we define the mapping $A \rightarrow A_{N, x}$ of $\mathscr{A}$ into $\mathscr{A}_{N}(\Omega)$ by the formula

$$
A_{N, x}=P_{N} \mathbf{E}\left(\sigma\left(N^{1 / 3}, x\right) A / \Omega\right) P_{N} .
$$

The $A_{N, x}$ 's correspond to observables for $\mathscr{G}_{N}$, localised around $x$, as represented in a scaling where the unit of length is $N^{-1 / 3}$, which is essentially the mean interparticle spacing.

Let $\mathscr{S}(\mathscr{A})$ be the set of all translationally invariant states on $\mathscr{A}$, and let $t, s, f\left(:=t-\beta^{-1} s\right)$ and $n$ denote the functionals on $\mathscr{S}(\mathscr{A})$, defined in [12], corresponding to the densities of kinetic energy, entropy, free energy and particle number, respectively, for the ideal Fermi gas, $\mathscr{I}$. The functionals $f$ and $n$ are thus affine and $\left(w^{*}-\right)$ lower semicontinuous. As will be proved in Appendix 2, $f$ has a unique minimum, $\bar{\omega}_{\varrho}$, on $\mathscr{S}(\mathscr{A}) \cap n^{-1}(\varrho)$, and

$$
f\left(\bar{\omega}_{\varrho}\right)=\varphi_{0}(\varrho) .
$$

Further, it may easily be inferred from the formulae in Appendix 2 that the $\operatorname{map} \varrho \rightarrow \bar{\omega}_{\varrho}$ is $w^{*}$-continuous; while $\varphi_{0}$ is lower-bounded, continuous, and bounded on the compacts, and tends to $\infty$ as $\varrho \rightarrow \infty$.

We are now in a position to state our main theorem concerning the limiting form, as $N \rightarrow \infty$, of the equilibrium correlation functions for the re-scaled observables $\left\{A_{N, x}\right\}$ of $\mathscr{G}_{N}$.

Theorem 1. If the Thomas-Fermi functional $\Phi_{0}$ is minimised at a unique bounded probability density $\varrho_{0}$ on $\Omega$, then

$$
\begin{aligned}
& \lim _{N \rightarrow \infty} \int d^{3} x_{1} \ldots d^{3} x_{k} \omega_{N}\left(\prod_{1}^{k} A_{N, x_{2}}^{(i)}\right) h\left(x_{1}, \ldots, x_{k}\right) \\
& \quad=\int d^{3} x_{1} \ldots d^{3} x_{k}\left(\prod_{1}^{k} \bar{\omega}_{\varrho_{0}\left(x_{i}\right)}\left(A^{(i)}\right)\right) h\left(x_{1}, \ldots, x_{k}\right), \\
& \forall A^{(1)}, \ldots, A^{(k)} \in \mathscr{A}: h \in \mathscr{B} \mathscr{C}\left(\Omega^{k}\right),
\end{aligned}
$$


where $\Pi_{+}$denotes symmetrised product and $\mathscr{B} \mathscr{C}\left(\Omega^{k}\right)$ is the set of bounded continuous functions on $\Omega^{k}$.

We define $\mathscr{H} \mathscr{L}(\mathscr{A})$, the hydrolocal algebra, to be the discrete tensor product $\bigotimes_{x \in \Omega} \mathscr{A}_{x}$, where the $\mathscr{A}_{x}$ 's are copies of $\mathscr{A}$. Thus, $\mathscr{H} \mathscr{L}(\mathscr{A})$ is the inductive limit of the $C^{*}$-tensor products $\bigotimes_{x \in F} \mathscr{A}_{x}$ over finite point subsets $F$ of $\Omega$, equipped with the canonical injection from $\bigotimes_{x \in F} \mathscr{A}_{x}$ into $\bigotimes_{x \in F^{\prime}} \mathscr{A}_{x}$ for $F \subset F^{\prime}$. For $A^{(1)}, \ldots, A^{(k)} \in \mathscr{A}$ and $x_{1}, \ldots x_{k}$ different points of $\Omega$, we define $\left[A^{(1)}, \ldots A^{(k)} ; x_{1}, \ldots x_{k}\right]$ to be element of $\mathscr{H} \mathscr{L}(\mathscr{A})$ given by $\bigotimes_{x \in \Omega} A_{x}$, with $A_{x_{i}}=A^{(i)}$ for $i=1, \ldots k$ and $A_{x}=I$ for $x \notin\left\{x_{1}, \ldots, x_{k}\right\}$. We then define $J_{N}$ to be the linear mapping, from $\mathscr{H} \mathscr{L}(\mathscr{A})$ into the bounded operators in $\mathscr{H}_{N}(\Omega)$, by the formula

$$
J_{N}\left[A^{(1)}, \ldots, A^{(k)} ; x_{1}, \ldots, x_{k}\right]=\prod_{i=1}^{k} A_{N, x_{i}}^{(i)} .
$$

We see immediately from these definitions that Theorem 1 may be restated in the following form.

Theorem 1'. If the Thomas-Fermi functional $\Phi_{0}$ is minimised at a unique bounded probability density $\varrho_{0}$ on $\Omega$, then

$$
\begin{aligned}
& \lim _{N \rightarrow \infty} \int d^{3} x_{1} \ldots d^{3} x_{k}\left(\omega_{N} J_{N}\right)\left(\left[A^{(1)}, \ldots, A^{(k)} ; x_{1}, \ldots, x_{k}\right]\right) h\left(x_{1}, \ldots, x_{k}\right) \\
& \quad=\int d^{3} x_{1} \ldots d^{3} x_{k} \bar{\omega}\left(\left[A^{(1)}, \ldots, A^{(k)} ; x_{1}, \ldots, x_{k}\right]\right) h\left(x_{1}, \ldots, x_{k}\right) \\
& \forall A^{(1)}, \ldots, A^{(k)} \in \mathscr{A} ; h \in \mathscr{B} \mathscr{C}\left(\Omega^{k}\right) ; k<\infty,
\end{aligned}
$$

where

$$
\bar{\omega}:=\bigotimes_{x \in \Omega} \bar{\omega}_{\varrho_{0}(x)} .
$$

\section{Comments}

1. According to the numerical analysis of the Thomas-Fermi Euler equation, $\delta \Phi_{0} / \delta \varrho(x)=0$, for the case where $\Omega$ is spherical, the functional $\Phi_{0}$ is minimised at a unique bounded probability density $\varrho_{0}$, except at the critical temperature $T_{c}$. Accepting this result, we see that the condition governing Theorems 1 and $1^{\prime}$ is fulfilled, at least when $\Omega$ is spherical and $T \neq T_{c}$.

2. Theorem 1' specifies a precise sense in which $\bar{\omega}$ is the limiting form of $\omega_{N} \circ J_{N}$ as $N \rightarrow \infty$. We interpret this theorem as signifying that the state $\bar{\omega}$ on the hydrolocal algebra $\mathscr{H} \mathscr{L}(\mathscr{A})$ represents the properties of $\omega_{N}$ in the limit $N \rightarrow \infty$.

3. We propose that $\bar{\omega}$ be taken to be an equilibrium state of the infinite system, not only because it corresponds to the limit of a sequence of Gibbs states, but also because it has the following stability properties.

(a) $\bar{\omega}$ is globally stable, in the sense that its specific free energy is the minimum value of the Thomas-Fermi functional $\Phi_{0}$.

(b) $\bar{\omega}$ is stable at the strictly local level, in the sense that its components $\bar{\omega}_{\varrho_{0}(x)}$ at the points $x$ of $\Omega$ are equilibrium states for a Fermi gas with the prevailing local density $\varrho_{0}(x)$, the value of which is determined by the minimisation of $\Phi_{0}$. 
4. We conjecture that the system may also possess metastable ${ }^{1}$ states for the following reason. According to the numerical treatment of [3], the Euler equation $\left(\delta \Phi_{0} / \delta \varrho(x)=0\right)$ governing the densities at which $\Phi_{0}$ is stationary, has solutions other than $\varrho_{0}$ when $\beta$ exceeds a critical value $\beta_{c}$; and one of these solutions, $\varrho_{1}$, corresponds to a smooth continuation, in $\beta$, of $\varrho_{0}$ from the region $\beta<\beta_{c}$. Accepting this result, one sees that $\bar{\omega}_{1}:=\bigotimes_{x \in \Omega} \bar{\omega}_{\varrho_{1}(x)}$ might be a candidate for a metastable state, satisfying criteria specified in [7], since on the one hand it lacks the global stability of (3a), while on the other it possesses the strictly local stability of (3b). In order to establish $\bar{\omega}_{1}$ as a metastable state, it would be necessary, in our view, to show firstly that it corresponds to the limit, analogous to that of Eq. (2.11), of a sequence of Gibbs states for the $N$-particle systems $\mathscr{G}_{N}$ whose densities are subjected to appropriate constraints; and secondly to prove that $\varrho_{1}$ is the absolute minimum of the restriction of $\Phi_{0}$ to the resultant constrained set of density functions. If these properties were established, then it would follow that $\bar{\omega}_{1}$ would be stable at both the strictly local and the local hydrodynamical levels, though not at the global one, and would thus be metastable in a sense that slightly generalises that prescribed in [7].

\section{The Perturbed System}

Our strategy for proving Theorem 1 will be centred on a treatment of the response of the system $\mathscr{G}_{N}$ to a certain class of perturbations. Thus, we start by defining the perturbed Hamiltonian

$$
H_{N}(\lambda)=H_{N}+\lambda \int_{\Omega^{k}} d^{3} x_{1} \ldots d^{3} x_{k} h\left(x_{1}, \ldots, x_{k}\right) \prod_{i=1}^{k} A_{N, x_{i}}^{(i)}
$$

where $\lambda \in \mathbf{R}, h \in \mathscr{B} \mathscr{C}\left(\Omega^{k}\right)$ and $A^{(1)}, \ldots A^{(k)}$ are self-adjoint elements of $\mathscr{A}_{L}$. The specific free energy of the perturbed system is then

$$
F_{N}(\lambda)=-(N \beta)^{-1} \ln \operatorname{Tr} \exp -\beta H_{N}(\lambda)
$$

from which one sees that $F_{N}$ is a concave function $\lambda$. By Eqs. (2.3), (3.1) and (3.2),

$$
F_{N}^{\prime}(0)=\int d^{3} x_{1} \ldots d^{3} x_{k} h\left(x_{1}, \ldots, x_{k}\right) \omega_{N}\left(\prod_{i=1}^{k}+A_{N, x_{i}}^{(i)}\right)
$$

Hence, $F_{N}^{\prime}(0)$ is equal to the value of the expression on the L.H.S. of Eq. (2.9) before the limit $N \rightarrow \infty$ is taken.

In order to relate the function $F_{N}$ to properties of the ideal Fermi gas, $\mathscr{I}$, we define $\Delta$ to be the subset of $\mathbf{R}_{+} \times \mathbf{R}^{k}$ given by

$$
\begin{aligned}
& \left\{(\varrho, \alpha) \mid \varrho \in R_{+} ; \alpha=\left(\alpha^{(1)}, \ldots, \alpha^{(k)}\right) \in \mathbf{R}^{k} ; \exists \omega \in \mathscr{S}(\mathscr{A}) .\right. \\
& \left.\quad f(\omega)<\infty ; n(\omega)=\varrho ; \omega\left(A^{(1)}\right)=\alpha^{(i)}, \text { for } i=1, \ldots, k\right\} ;
\end{aligned}
$$

1 The suggestion that the model may possess metastable states was first made to us by W. Thirring 
and we define $\varphi: \mathbf{R}_{+} \times \mathbf{R}^{k} \rightarrow \mathbf{R} \cup\{\infty\}$ by the formula

$$
\varphi(\varrho, \alpha)=\left\{\begin{array}{l}
\inf \left\{f(\omega) \mid \omega \in \mathscr{S}(\mathscr{A}) ; n(\omega)=\varrho ; \omega\left(A^{(i)}\right)=\alpha^{(i)}, i=1, \ldots, k\right\} \\
\text { if }(\varrho, \alpha) \in \Delta ; \\
\text { and }=\infty \text { otherwise } .
\end{array}\right.
$$

Thus, as the functionals $n$ and $f$ are affine [12], it follows that $\Delta$ is a convex set and that $\varphi$ is jointly convex in its arguments. We define $\hat{\varphi}$ to be the closure of $\varphi$, i.e. the greatest lower semi-continuous function on $\mathbf{R}_{+} \times \mathbf{R}^{k}$ that is majorised by $\varphi$ [13]: $\hat{\varphi}$ is thus also jointly convex in its arguments.

Let $T$ be the space of $L_{\infty}$-class functions on $\Omega$, equipped with the $w^{*}$-topology dual to the bounded continuous functions on that space. We define $\Theta$ to be the subspace of $T^{k+1}$ given by $\left\{\theta=(\varrho, \alpha) \mid \varrho \in L_{\infty}(\Omega) ; \alpha \in L_{\infty}(\Omega)^{k} ; \varrho>0 ; \int d^{3} x \varrho(x)=1\right\}$; and for $B \in \mathbf{R}$, we define $\Theta^{(B)}$ to be the subspace of $\Theta$ given by $\{\theta \in \Theta \mid \hat{\varphi}(\theta(x)) \leqq B$ for $x$ a.e. in $\Omega$. We then define the generalised Thomas-Fermi functional $\hat{\Phi}_{\lambda}$ on $\Theta$ by the following equations.

$$
\hat{\Phi}_{\lambda}=\hat{\Phi}+\lambda \Psi
$$

where

$$
\hat{\Phi}(\varrho, \alpha)=\int_{\Omega} d^{3} x \hat{\varphi}(\varrho(x), \alpha(x))+\frac{1}{2} \int_{\Omega^{2}} d^{3} x d^{3} y v(x, y) \varrho(x) \varrho(y)
$$

and

$$
\Psi(\varrho, \alpha)=\int_{\Omega^{k}} d^{3} x_{1} \ldots d^{3} x_{k} h\left(x_{1}, \ldots, x_{k}\right) \alpha^{(1)}\left(x_{1}\right) \ldots \alpha^{(k)}\left(x_{k}\right)
$$

We now see from Eqs. (3.3) and (3.7) that Theorem 1 is an immediate consequence of the following Lemma 2 and Theorem 3 and 4.

Lemma 2 [14]. If $\left\{f_{n}\right\}$ is a sequence of real-valued concave functions on $R$ converging pointwise to $f$, and if $f_{n}$ and $f$ are differentiable at $t(\in \mathbf{R})$, then

$$
\lim _{n \rightarrow \infty} f_{n}^{\prime}(t)=f^{\prime}(t) \text {. }
$$

Theorem 3. Given $\lambda_{0} \in \mathbf{R}_{+}, \exists B_{0} \in \mathbf{R}_{+}$such that, for all $|\lambda|<\lambda_{0}$, and for arbitrary $B>B_{0}$,

$$
\lim _{N \rightarrow \infty} F_{N}(\lambda)=\min \left\{\hat{\Phi}_{\lambda}(\varrho, \alpha) \mid(\varrho, \alpha) \in \Theta^{(B)}\right\}:=F(\lambda) .
$$

Theorem 4. If the Thomas-Fermi functional $\Phi_{0}$ is minimised at the unique bounded probability density $\varrho_{0}$ on $\Omega$, then

$$
F^{\prime}(0)=\Psi\left(\varrho_{0}, \alpha_{0}\right),
$$

where

$$
\alpha_{0}(x) \equiv\left(\alpha_{0}^{(1)}(\lambda), \ldots, \alpha_{0}^{(k)}(x)\right) ; \quad \text { and } \quad \alpha_{0}^{(i)}(x)=\bar{\omega}_{\varrho_{0}(x)}\left(A^{(i)}\right) .
$$

We conclude this section with the statement of the following lemmas, that will be used in the proofs of Theorems 3 and 4 . 
Lemma 5. If $\Phi_{0}$ is minimised at the unique bounded probability density $\varrho_{0}$, then $\hat{\Phi}$ is minimised at $\left(\varrho_{0}, \alpha_{0}\right)$ uniquely, where $\alpha_{0}$ is defined by Eq. (3.10); and further

$$
\hat{\varphi}\left(\varrho_{0}(x), \alpha_{0}(x)\right)=\varphi_{0}\left(\varrho_{0}(x)\right)
$$

Lemma 6. For $B \in \mathbf{R}, \Theta^{(B)}$ is a complete, compact, metrisable space; and there exists a finite $\bar{\varrho}_{B}$ such that if $(\varrho, \alpha) \in \Theta^{(B)}$, then $\|\varrho\|_{\infty}<\bar{\varrho}_{B}$ and $\left\|\alpha^{(i)}\right\|_{\infty}<\left\|A^{(i)}\right\|$ for $i=1, \ldots k$.

\section{Constructions}

In order to establish Theorem 4, we shall now make a number of constructions, similar to those of [4]. These constructions will be carried out explicitly for the case where $\Omega$ is a cube of side $l$. We note here that the restriction to such a form for $\Omega$ is quite inessential as the same results would be obtained, with slightly lengthier arguments, for any domain that is sufficiently regular to be approximated arbitrarily closely by unions of 'small' cubes. In the following analysis, we shall make the dependence of $F_{N}(\lambda)$ on $\beta$ and $l$ explicit, where necessary, denoting this quantity by $F_{N}(\lambda, \beta, l)$.

\section{(i) Regularisation of the Potential}

We approximate the Newtonian potential $v$ by a regular one $v_{\mu}$, defined by the formula

$$
v_{\mu}(x, y)=-\frac{(1-\exp -\mu|x-y|)}{|x-y|},
$$

with $\mu>0$; and we define $H_{N \mu}(\lambda)$ and $F_{N \mu}(\lambda, \beta, l)$ to be the Hamiltonian and specific free energy, respectively, resulting from the replacement of $v$ by $v_{\mu}$ in (2.1) and (2.2). On following the procedure of [4: Sect. 3$]$, we find that

$$
\begin{aligned}
& \left(1+2 \mu^{-1 / 5}\right)^{-1} F_{N \mu}\left(\lambda\left(1+2 \mu^{-1 / 5}\right), \beta\left(1+2 \mu^{-1 / 5}\right)^{-1}, l\right)-b_{1}(N, \mu) \\
& \quad \leqq F_{N}(\lambda, \beta, l) \leqq F_{N \mu}(\lambda, \beta, l)
\end{aligned}
$$

where

$$
\lim _{\mu \rightarrow \infty} \lim _{N \rightarrow \infty} b_{1}(N, \mu)=0 .
$$

\section{(ii) Division of $\Omega$ into Cells}

We divide $\Omega$ into $g$ equal cubic cells $C_{1}, \ldots C_{g}$, centred at $\left(c_{1}, \ldots c_{g}\right)$, respectively, and separated by partitions. We then introduce the following three operations that change the Hamiltonian from $H_{N \mu}(\lambda)$ to $H_{N \mu g}(\lambda)$.

(a) We impose Dirichlet boundary conditions at the boundaries of the cells so as to represent the presence of the partitions.

(b) We replace $v_{\mu}$ by the step-function $v_{\mu g}$, where

$$
v_{\mu g}(x, y)=\left\{\begin{array}{l}
v_{\mu}\left(c_{r}, c_{s}\right) \text { if } x \in C_{r}, y \in C_{s}, r \neq s \\
0 \text { if } x, y \text { lie in the same cell. }
\end{array}\right.
$$


(c) For each of the cells $C_{r}$, we define $C_{r}^{(N)}$ to be the largest open cube in $C_{r}$ such that, for $x \in C_{r}^{(N)}, \sigma\left(N^{1 / 3}, x\right) A^{(i)} \in \mathscr{A}\left(C_{r}\right)$ for $r=1, \ldots, g$. We then replace $h$ by $h_{g} \mathscr{X}_{g}^{(N)}$, where $\mathscr{X}_{g}^{(N)}$ is the characteristic function for $\left(\bigcup_{r=1}^{g} C_{r}^{(N)}\right)^{k}$, and $h_{g}$ is the step-function given by the formula

$$
h_{g}\left(x_{1}, \ldots, x_{k}\right)=\left\{\begin{array}{l}
h\left(c_{r_{1}}, \ldots, c_{r_{k}}\right) \text { if } \quad x_{i} \in C_{r_{i}} ; i=1, \ldots, k ; r_{i} \neq r_{j} \text { for } i \neq j \\
0 \quad \text { otherwise. }
\end{array}\right.
$$

We note here that it follows from our definition of $C_{r}^{(N)}$, together with Eq. (2.5), that

$$
\lim _{N \rightarrow \infty}\left|C_{r}^{(N)}\right| /\left|C_{r}\right|=1 \text {. }
$$

On following the procedure of [4: Sect. 4], we obtain the following estimate for the specific free energy $F_{N \mu g}(\lambda)$, corresponding to the Hamiltonian $H_{N \mu g}(\lambda)$.

$$
\begin{aligned}
& F_{N \mu g}\left(\lambda, \beta, l+b_{2}(g)\right)-b_{3}(N, \mu, g) \leqq F_{N \mu}(\lambda, \beta, l) \\
& \quad \leqq F_{N \mu g}(\lambda, \beta, l)+b_{3}(N, \mu, g),
\end{aligned}
$$

where

$$
b_{2}(g)>0 ; \lim _{g \rightarrow \infty} b_{2}(g)=0 ; \text { and } \lim _{g \rightarrow \infty} \lim _{N \rightarrow \infty} b_{3}(N, \mu, g)=0 .
$$

\section{(iii) Distribution of Particles Among the Cells}

The separation of the cells by partitions restricts the particle configurations in such a way that the number of particles in each cell is an integer. Accordingly, the set of admissible distributions of particles among the cells corresponds to $P_{N}:=\left\{\varrho=\left(\varrho_{1}, \ldots, \varrho_{g}\right)\left|N \varrho_{r}\right| C_{r} \in Z_{+}\right.$for $\left.r=1, \ldots, g ; \sum_{r=1}^{g} \varrho_{r}\left|C_{r}\right|=1\right\}:$ the component $\varrho_{r}$ of $\varrho\left(\in P_{N}\right)$ then corresponds to $N^{-1} \times$ mean particle density for $C_{r}$. For $\varrho \in P_{N}$, we define $F_{N \mu g e}(\lambda, \beta, l)$ to be the specific free energy of the system with Hamiltonian $H_{N \mu g}(\lambda)$, subject to the constraint that the distribution of particles among the cells is given by $\varrho$. We define

$$
\bar{F}_{N \mu g}(\lambda, \beta, l):=\min _{\varrho \in P_{N}} F_{N \mu g e}(\lambda, \beta, l),
$$

and, by a simple extension of the argument of [4, Sect. 5], we find that

$$
\lim _{N \rightarrow \infty}\left[\bar{F}_{N \mu g}(\lambda, \beta, l)-F_{N \mu g}(\lambda, \beta, l)\right]=0,
$$

\section{(iv) Thomas-Fermi Functionals}

Let $\hat{\Phi}_{\mu \lambda}$ be the functional obtained by replacing $v$ by $v_{\mu}$, and let $\hat{\Phi}_{\mu g \lambda}$ be the one obtained by replacing $v, h$ by $v_{\mu g}, h_{g}$ in the formulae (3.5)-(3.7), that define $\hat{\Phi}_{\lambda}$.

Lemma 7. The restrictions of $\hat{\Phi}_{\lambda}$ and $\hat{\Phi}_{\mu \lambda}$ to $\Theta^{(B)}$ are lower semicontinuous.

Lemma 8. Let $\Theta_{g}$ be the subset of $\Theta$ whose elements $\Theta$ take uniform values in each of the cells $C_{1}, \ldots C_{g}$. Then, given $\lambda_{0} \in \mathbf{R}_{+}$, and intervals $\left(\beta_{1}, \beta_{2}\right),\left(l_{1}, l_{2}\right)$ on the positive 
real line, there exists $B_{0} \in \mathbf{R}$ such that, for $\mu, g \in \mathbf{R}_{+}, \beta \in\left(\beta_{1}, \beta_{2}\right), l \in\left(l_{1}, l_{2}\right)$ and $|\lambda|<\lambda_{0}$, the restriction of $\hat{\Phi}_{\mu g \lambda}$ to $\Theta_{g}$ is minimised at an element $\theta_{\mu g \lambda}$ of $\Theta^{\left(B_{0}\right)}$.

\section{Theorem 9.}

$$
\begin{aligned}
\lim _{N \rightarrow \infty} F_{N \mu g}(\lambda, \beta, l) & =\min \left\{\hat{\Phi}_{\mu g \lambda}(\varrho, \alpha) \mid(\varrho, \alpha) \in \Theta_{g}\right\} \\
: & =F_{\mu g}(\lambda, \beta, l)
\end{aligned}
$$

Theorem 10. With the same specifications for $\lambda_{0}$ and $B_{0}$ as in Lemma 8, and for arbitrary $B>B_{0}$,

$$
\begin{gathered}
\lim _{\mu \rightarrow \infty} \lim _{g \rightarrow \infty} F_{\mu g}(\lambda, \beta, l)=\min \left\{\hat{\Phi}_{\lambda}(\varrho, \alpha) \mid(\varrho, \alpha) \in \Theta^{(B)}\right\} \\
:=F(\lambda, \beta, l) \forall|\lambda|<\lambda_{0}
\end{gathered}
$$

\section{Theorem 11.}

$$
\lim _{N \rightarrow \infty} F_{N}(\lambda, \beta, l)=F(\lambda, \beta, l) \forall|\lambda|<\lambda_{0} .
$$

\section{Proof of the Theorems}

As already noted, Theorem 1 follows directly from Lemma 2 and Theorems 3 and 4. Further, Theorem 3 is an immediate consequence of Theorems 10 and 11. Hence, the only theorems for which proof is needed are Theorems 4, 9, 10 and 11.

Proof of Theorem 4. Assuming that $\Phi_{0}$ is minimised at $\varrho_{0}$, uniquely, it follows from Lemma 5 that $\hat{\Phi}$ is minimised at $\theta_{0}:=\left(\varrho_{0}, \alpha_{0}\right)$ uniquely; and that, as $\varrho_{0} \in L_{\infty}(\Omega)$ and as $\varphi_{0}$ is bounded on the compacts, then in view of Eq. (3.11), $\left\|\hat{\varphi} \circ \theta_{0}\right\|_{\infty}<\infty$. Let $\lambda_{0} \in \mathbf{R}_{+}$, let $B_{0}(\in \mathbf{R})$ be specified as in Theorem 3 and choose $B$ to be some real number that exceeds both $B_{0}$ and $\left\|\hat{\varphi} \circ \theta_{0}\right\|_{\infty}$, thereby ensuring that $\theta_{0} \in \Theta^{(B)}$ and that Eq. (3.8) is applicable for $|\lambda|<\lambda_{0}$. Thus, if $\theta_{\lambda}$ is an element of $\Theta^{(B)}$ at which $\hat{\Phi}_{\lambda}$ is minimised, then

$$
F(\lambda)=\hat{\Phi}_{\lambda}\left(\theta_{\lambda}\right) \leqq \hat{\Phi}_{\lambda}\left(\theta_{0}\right)
$$

and

$$
F(0)=\hat{\Phi}\left(\theta_{0}\right) \leqq \hat{\Phi}\left(\theta_{\lambda}\right)
$$

Further since, by Eq. (3.7) and Lemma 6, one can find $k<\infty$ such that $|\Psi(\theta)|<k|\lambda| \forall \theta \in \Theta^{(B)}$, it follows from Eqs. (3.5), (5.1) and (5.2) that $\left|\hat{\Phi}\left(\theta_{\lambda}\right)-\hat{\Phi}\left(\theta_{0}\right)\right|<2 k|\lambda|$ and therefore

$$
\lim _{\lambda \rightarrow \infty} \hat{\Phi}\left(\theta_{\lambda}\right)=\hat{\Phi}\left(\theta_{0}\right) \text {. }
$$

On the other hand, as $\Theta^{(B)}$ is a compact, metrisable space, by Lemma 6 , one can choose a sequence of positive numbers $\left\{\lambda_{n}\right\}$, tending to zero such that $\theta_{\lambda_{n}}$ converges to an element $\theta_{0}^{\prime}$, say, of $\Theta^{(B)}$. Hence, as $\hat{\Phi}$ is lower semi-continuous, by Lemma 7, it follows from Eq. (5.3) that $\hat{\Phi}\left(\theta_{0}^{\prime}\right) \leqq \hat{\Phi}\left(\theta_{0}\right)$; and therefore $\theta_{0}^{\prime}=\theta_{0}$, as $\hat{\Phi}$ is minimised at $\theta_{0}$ uniquely. Thus

$$
\lim _{n \rightarrow \infty} \theta_{\lambda_{n}}=\theta_{0} \text {. }
$$


As $F_{N}$ is a concave function of $\lambda$, we see from Eq. (3.8) that so too is $F$. We denote its left and right derivatives by $F_{l}^{\prime}$ and $F_{r}^{\prime}$, respectively. By Eqs. (3.5), (5.1) and (5.2),

$$
\frac{F\left(\lambda_{n}\right)-F(0)}{\lambda_{n}}=\frac{\hat{\Phi}\left(\theta_{\lambda_{n}}\right)-\hat{\Phi}\left(\theta_{0}\right)}{\lambda_{n}}+\Psi\left(\theta_{\lambda_{n}}\right) \geqq \Psi\left(\theta_{\lambda_{n}}\right)
$$

from which it follows that

$$
F_{r}^{\prime}(0) \geqq \limsup _{n \rightarrow \infty} \Psi\left(\theta_{\lambda_{n}}\right) .
$$

Moreover, it follows easily from Eq. (3.7) that the functional $\Psi$ is continuous, and therefore by (5.4) and (5.5),

$$
F_{r}^{\prime}(0) \geqq \Psi\left(\theta_{0}\right)
$$

Similarly by considering a sequence $\left\{\theta_{\lambda_{n}}\right\}$ of elements of $\Theta^{(B)}$ corresponding to negative numbers $\left\{\lambda_{n}\right\}$, one finds that

$$
F_{l}^{\prime}(0) \leqq \Psi\left(\theta_{0}\right)
$$

Since $F$ is concave, it follows immediately from (5.6) and (5.7) that this function is differentiably at $\lambda=0$, and that $F^{\prime}(0)=\Psi\left(\theta_{0}\right)$.

Proof of Theorem 9. Let $\mathscr{G}_{N, \varrho}$ be the system of $N$ gravitating particles, whose distribution among the cells $C_{1}, \ldots C_{g}$ is given by $\varrho\left(\in P_{N}\right)$. The normal states of $\mathscr{G}_{N, \varrho}$ correspond to density matrices in $\mathscr{H}_{N, \varrho}:=\bigotimes_{r=1}^{g} \mathscr{H}_{N_{r}}\left(C_{r}\right)$, where $\mathscr{H}_{N_{r}}\left(C_{r}\right)$ is the $N_{r^{-}}$ particle subspace of the Fock space $\mathscr{H}\left(C_{r}\right)$, and $N_{r}=N \varrho_{r}\left|C_{r}\right|$. In formulating $\mathscr{G}_{N, \varrho}$, we shall generally use the same symbol to denote an operator in $\mathscr{H}_{N_{r}}\left(C_{r}\right)$ and its canonical injection into $\mathscr{H}_{N, \varrho}$.

The Hamiltonian $H_{N \mu g \varrho}(\lambda)$ for $\mathscr{G}_{N, \varrho}$, corresponding to the truncated interactions $v_{\mu g}$ and $h_{g}$ specified in Sect. 4 , is simply the restriction of $H_{N \mu g}(\lambda)$ to $\mathscr{H}_{N, \varrho}$. Thus

$$
\begin{aligned}
H_{N \mu g \varrho}(\lambda)= & N^{-2 / 3} \sum_{r=1}^{g} \mathrm{~T}_{r}+\frac{1}{2} N \sum_{r, s=1}^{g} v_{r s} \varrho_{r} \varrho_{s}\left|C_{r}\right|\left|C_{s}\right| \\
& +\lambda N \sum_{r_{1}, \ldots, r_{k}=1}^{g} h_{r_{1}} \ldots r_{k} A_{r_{1}}^{(1)} \ldots A_{r_{k}}^{(k)}\left(C_{r_{1}}|\ldots| C_{r_{k}} \mid\right.
\end{aligned}
$$

where $T_{r}$ corresponds to the operator in $\mathscr{H}_{N_{r}}\left(C_{r}\right)$ representing the kinetic energy of $N_{r}$ particles of unit mass in $C_{r}$, i.e.

$$
\mathrm{T}_{r}=-\frac{1}{2} \sum_{j=1}^{N_{r}} \Delta_{j}
$$

where Dirichlet boundary conditions are imposed; $A_{r}^{(i)}$ corresponds to the operator in $\mathscr{H}_{N_{r}}\left(C_{r}\right)$ given by

$$
\begin{aligned}
A_{r}^{(i)} & =\left|C_{r}\right|^{-1} \int_{C_{r}^{(N)}} d^{3} x\left(\sigma\left(N^{1 / 3}, x\right) A^{(i)}\right)_{N_{r}} ; \\
v_{r s} & =v_{\mu g}\left(c_{r}, c_{s}\right),
\end{aligned}
$$


and

$$
h_{r_{1} \ldots r_{k}}=h_{g}\left(c_{r_{1}}, \ldots, c_{r_{k}}\right) .
$$

Correspondingly, the specific free energy of $\mathscr{G}_{N, \varrho}$ is

$$
F_{N \mu g \varrho}(\lambda)=-(N \beta)^{-1} \ln \operatorname{Tr} \exp \left(-\beta H_{N \mu g \varrho}(\lambda)\right),
$$

the trace being taken over $\mathscr{H}_{N, \varrho}$.

In order that we may apply standard thermodynamical limiting procedures to this formula, we now cast it into a form that expresses $F_{N \mu g \varrho}(\lambda)$ as the specific free energy of an $N$-particle system occupying a volume proportional to $N$. To this end, we define $\tilde{C}_{r}:=N^{1 / 3} C_{r}, \tilde{\mathscr{H}}_{N, \varrho}:=\bigotimes_{r=1}^{g} \mathscr{H}_{N_{r}}\left(\tilde{C}_{r}\right)$ and $\tilde{H}_{N \mu g \varrho}(\lambda)$ to be the operator in $\tilde{\mathscr{H}}_{N, \varrho}$ given by

$$
\tilde{H}_{N \mu g \varrho}(\lambda):=U\left(N^{1 / 3}, 0\right)^{-1} H_{N \mu g \varrho}(\lambda) U\left(N^{1 / 3}, 0\right),
$$

where $U$ is defined in Eq. (2.6). Thus, by Eqs. (2.6), (5.8)-(5.10), and (5.14), it follows that

$$
\begin{aligned}
\tilde{H}_{N \mu g Q}(\lambda)= & \sum_{r=1}^{g} \tilde{T}_{r}+\frac{1}{2} N \sum_{r, s=1}^{g} v_{r s} \varrho_{r} \varrho_{s} \\
& +\lambda N \sum_{r_{1}, \ldots, r_{k}=1}^{g} h_{r_{1} \ldots r_{k}} \tilde{A}_{r_{1}}^{(1)} \ldots \tilde{A}_{r_{k}}^{(k)}\left|C_{r_{1}}\right| \ldots\left|C_{r_{k}}\right|
\end{aligned}
$$

where $\tilde{T}_{r}$ is the kinetic energy operator for $N_{r}$ particles of unit-mass in $C_{r}$, with Dirichlet boundary conditions,

$$
\left.\tilde{A}_{r}^{(i)}:=\left|\tilde{C}_{r}\right|^{-1} \int_{C_{r}^{(N)}} d^{3} x(\tau(x)) A^{(i)}\right)_{N_{r}},
$$

$\tau\left(R^{3}\right)$ is the group of automorphisms of $\mathscr{A}$ corresponding to space translations, and $\tilde{C}_{r}^{(N)}:=N^{1 / 3} C_{r}^{(N)}$. Thus, in view of Eq. (4.6),

$$
\lim _{N \rightarrow \infty}\left|\tilde{C}_{r}^{(N)}\right| /\left|\tilde{C}_{r}\right|=1 \text {. }
$$

It follows immediately from Eqs. (5.13), (5.14) and the unitarity of $U$ that

$$
F_{N \mu g \varrho}(\lambda)=-(N \beta)^{-1} \ln \tilde{\operatorname{Tr}} \exp \left(-\beta \tilde{H}_{N \mu g \varrho}(\lambda)\right),
$$

where $\tilde{T}$ r denotes the trace over $\tilde{\mathscr{H}}_{N, \varrho}$; and hence, by (4.9).

$$
\bar{F}_{N \mu g}(\lambda, \beta, l)=\min _{\varrho \in P_{N}}\left[-(N \beta)^{-1} \ln \tilde{T} \operatorname{rexp}\left(-\beta \tilde{H}_{N \mu g \varrho}(\lambda)\right)\right] .
$$

This formula will be treated in Appendix 1, where it will be shown, by an extension of the methods of [8], that the space-averaged observables $\tilde{A}_{r}^{(i)}$ occurring in the formula for $\tilde{H}_{N \mu g \varrho}(\lambda)$ may be replaced by $c$-numbers satisfying a certain variational principle in the formula for $\bar{F}_{N \mu g}$, in the limit $N \rightarrow \infty$, with the result that

$$
\begin{aligned}
\lim _{N \rightarrow \infty} \bar{F}_{N \mu g}(\lambda, \beta, l)= & \min \left\{\sum_{r=1}^{g} \hat{\varphi}\left(\varrho_{r}, \alpha_{r}\right)\left|C_{r}\right|+\frac{1}{2} \sum_{r, s=1}^{g} v_{r s} \varrho_{r} \varrho_{s}\left|C_{r}\right|\left|C_{s}\right|\right. \\
& +\sum_{r_{1}, \ldots, r_{k}=1}^{g} h_{r_{1} \ldots r_{k}} \alpha_{r_{1}}^{(1)} \ldots \alpha_{r_{k}}^{(k)}\left|C_{r_{1}}\right| \ldots\left|C_{r_{k}}\right| \\
& \left.\left.\left(\varrho_{r}, \alpha_{r}\right) \in \mathbf{R}_{+} \times \mathbf{R}^{k} ; \sum_{1}^{g} \varrho_{r} \mid C_{r}\right)=1\right\} .
\end{aligned}
$$


This effectively completes the proof of the theorem, since the definitions of $\Theta_{g}$ and $\Phi_{\mu g \lambda}$ (in Sect. 4, Pt. 4) imply that the R.H.S. of (5.20) is equal to $\min \left\{\hat{\Phi}_{\mu g \lambda}(\theta) \mid \theta \in \Theta_{g}\right\}$; while it follows from Eq. (4.10) that the L.H.S. of (5.20) is equal to $\lim _{N \rightarrow \infty} F_{N \mu g}(\lambda, \beta, l)$.

Proof of Theorem 10. It follows from Lemmas 6, 7 and Theorem 9 that for $|\lambda|<\lambda_{0}$ and $B>B_{0}$, one can find elements $\theta_{\lambda}, \theta_{\mu \lambda}, \theta_{\mu g \lambda}$ of $\Theta^{(B)}, \Theta^{(B)}$ and $\Theta^{(B)} \cap \Theta_{g}$ at which $\hat{\Phi}_{\lambda}, \hat{\Phi}_{\mu \lambda}, \hat{\Phi}_{\mu g \lambda}$, respectively, are minimised. Since, by Theorem $9, F_{\mu g}(\lambda, \beta, l)$ $=\hat{\Phi}_{\mu g \lambda}\left(\theta_{\mu g \lambda}\right)$, it suffices for us to show that

$$
\lim _{g \rightarrow \infty} \hat{\Phi}_{\mu g \lambda}\left(\theta_{\mu \lambda}\right)=\hat{\Phi}_{\mu \lambda}\left(\theta_{\mu \lambda}\right)
$$

and that

$$
\lim _{\mu \rightarrow \infty} \hat{\Phi}_{\mu \lambda}\left(\theta_{\mu \lambda}\right)=\hat{\Phi}_{\lambda}\left(\theta_{\lambda}\right) .
$$

Let $\theta_{\mu g \lambda}^{\prime}$ be the element of $\Theta_{g}$ obtained by replacing $\theta_{\mu \lambda}$ in each cell $C_{\boldsymbol{r}}$ by its mean value over that cell. Then it follows from the convexity of $\hat{\varphi}$ and our definition of $\Theta^{(B)}$ that $\theta_{\mu g \lambda}^{\prime} \in \Theta^{(B)} \cap \Theta_{g}$. Hence as $\theta_{\mu \lambda}, \theta_{\mu g \lambda}$ are elements of $\Theta^{(B)}$, $\Theta^{(B)} \cap \Theta_{g}$ at which $\hat{\Phi}_{\mu \lambda}, \hat{\Phi}_{\mu g \lambda}$, respectively, are minimised,

$$
\hat{\Phi}_{\mu \lambda}\left(\theta_{\mu \lambda}\right) \leqq \hat{\Phi}_{\mu \lambda}\left(\theta_{\mu g \lambda}\right)
$$

and

$$
\hat{\Phi}_{\mu g \lambda}\left(\theta_{\mu g \lambda}\right) \leqq \hat{\Phi}_{\mu g \lambda}\left(\theta_{\mu g \lambda}^{\prime}\right) .
$$

Further, it follows from our definitions of $\Theta^{(B)}, \hat{\Phi}_{\mu \lambda}, \hat{\Phi}_{\mu g \lambda}$ in Sect. 4 (iii) and (iv) that, in view of the convexity of $\hat{\varphi}$ and the uniform boundedness of the elements of $\Theta^{(B)}$ (by Lemma 7)

$$
\limsup _{g \rightarrow \infty} \hat{\Phi}_{\mu \lambda}\left(\theta_{\mu g \lambda}^{\prime}\right) \leqq \hat{\Phi}_{\mu \lambda}\left(\theta_{\mu \lambda}\right)
$$

and

$$
\hat{\Phi}_{\mu g \lambda}(\theta) \rightarrow \hat{\Phi}_{\mu \lambda}(\theta) \text {, uniformly w.r.t. } \theta \text { in } \Theta^{(B)} \text {, as } g \rightarrow \infty \text {. }
$$

Hence, by Eqs. (5.23)-(5.26),

$$
\begin{aligned}
\hat{\Phi}_{\mu \lambda}\left(\theta_{\mu \lambda}\right) & \leqq \liminf _{g \rightarrow \infty} \hat{\Phi}_{\mu \lambda}\left(\theta_{\mu g \lambda}\right) \leqq \limsup _{g \rightarrow \infty} \hat{\Phi}_{\mu \lambda}\left(\theta_{\mu g \lambda}\right) \\
& =\limsup _{g \rightarrow \infty} \hat{\Phi}_{\mu g \lambda}\left(\theta_{\mu g \lambda}\right) \leqq \limsup _{g \rightarrow \infty} \hat{\Phi}_{\mu g \lambda}\left(\theta_{\mu g \lambda}^{\prime}\right)=\limsup _{g \rightarrow \infty} \hat{\Phi}_{\mu \lambda}\left(\theta_{\mu g \lambda}^{\prime}\right) \leqq \hat{\Phi}_{\mu \lambda}\left(\theta_{\mu \lambda}\right),
\end{aligned}
$$

from which it follows that (5.21) is valid.

Finally it follows from Lemma 6 and our definitions of $\hat{\Phi}_{\lambda}, \hat{\Phi}_{\mu \lambda}$ and $\Theta^{(B)}$ that, for any $\theta \in \Theta^{(B)}$,

$$
\left.\left|\hat{\Phi}_{\lambda}(\theta)-\hat{\Phi}_{\mu \lambda}(\theta)\right| \leqq \bar{\varrho}_{B}^{2} \int_{\Omega^{2}} d^{3} x d^{3} y \exp (-\mu \mid x-y)\right) /|x-y| ;
$$

and therefore $\hat{\Phi}_{\mu \lambda}(\theta) \rightarrow \hat{\Phi}_{\lambda}(\theta)$, uniformly with respect to $\theta \in \Theta^{(B)}$, as $\mu \rightarrow \infty$. Equation (5.22) follows immediately from this result and the definitions of $\theta_{\lambda}, \theta_{\mu \lambda}$ as elements of $\Theta^{(B)}$ at which $\hat{\Phi}_{\lambda}, \hat{\Phi}_{\mu \lambda}$, respectively, are minimised. 
Proof of Theorem 11. Since $h$ is bounded, one can easily infer from Eqs. (3.1), (3.2) that $F_{N}(\lambda, \beta, l)$ is non-decreasing in $\beta$, non-increasing in $l$ and uniformly continuous in $\lambda$, for $|\lambda|<\lambda_{0}$. Hence, it follows from (4.2), (4.3), (4.7), (4.8) that, given $\delta, \varepsilon>0$, then for sufficiently large $\mu$ and $g$,

$$
\begin{aligned}
& F_{N \mu g}(\lambda, \beta, l)+c(N, \mu, g) \geqq F_{N}(\lambda, \beta, l) \\
& \quad \geqq\left(1+2 \mu^{-1 / 5}\right)^{-1} F_{N \mu g}(\lambda, \beta-\delta, l+\varepsilon)-\left(1+2 \mu^{-1 / 5}\right)^{-1} c(N, \mu, g)
\end{aligned}
$$

where

$$
\lim _{\mu \rightarrow \infty} \lim _{g \rightarrow \infty} \lim _{N \rightarrow \infty} c(N, \mu, g)=0 .
$$

By Theorems 9, 10 and the boundedness conditions obtained from Lemmas 6-8, it follows from (5.27) and (5.28) that

$$
F(\lambda, \beta, l) \geqq \limsup _{N \rightarrow \infty} F_{N}(\lambda, \beta, l) \geqq \liminf _{N \rightarrow \infty} F_{N}(\lambda, \beta, l) \geqq F(\lambda, \beta-\delta, l+\varepsilon) .
$$

Thus, as $\delta, \varepsilon$ are arbitrary positive numbers, it suffices for us to establish that

$$
\lim _{\varepsilon \rightarrow 0} \lim _{\delta \rightarrow 0} F(\lambda, \beta-\delta, l+\varepsilon)=F(\lambda, \beta, l),
$$

in order to infer the desired result from (5.29).

Now, as $F_{N \mu g}(\lambda, \beta, l)$ is concave in $\beta^{-1}$, it follows from Theorems 9,10 that the same is true for $F(\lambda, \beta, l)$. Further, by Lemma 6 and Theorem $10, F(\lambda, \beta, l)$ is bounded for finite $\beta^{-1}$, and hence as it is concave in this variable, it is continuous in $\beta$ over bounded intervals that exclude the origin. Hence

$$
\lim _{\delta \rightarrow 0} F(\lambda, \beta-\delta, l+\varepsilon)=F(\lambda, \beta, l+\varepsilon)
$$

In order to pass to the limit $\varepsilon \rightarrow 0$, we first note that, for $q>0$, a treatment, parallel to that leading to Theorem 10 , yields the result that

$$
\begin{aligned}
& \lim _{\mu \rightarrow \infty} \lim _{g \rightarrow \infty} \lim _{N \rightarrow \infty}-\left(q^{-1} N \beta\right)^{-1} \ln \operatorname{Tr} \exp \left(-\beta H_{N \mu g}(\lambda)\right) \\
& \quad=\min \left\{\hat{\Phi}_{\lambda}(\varrho, \alpha) \mid \varrho \in L_{\infty}(\Omega) ; \alpha \in L_{\infty}(\Omega)^{k} ; \varrho>0 ; \int_{\Omega} d^{3} n \varrho(x)=q\right\} .
\end{aligned}
$$

The L.H.S. of this equation may be seen from Theorems 9, 10, and our definition of $F_{N \mu g}$ to be $q F(\lambda, \beta, l)$. Hence it follows from Eq. (4.12) that

$$
q F(\lambda, \beta, l) \leqq \hat{\Phi}_{\lambda}(\varrho, \alpha) \quad \text { if } \quad \varrho \in L_{\infty}(\Omega), \alpha \in L_{\infty}(\Omega)^{k}, \varrho>0, \int_{\Omega} d^{3} x \varrho(x)=q .
$$

Now let $\Omega_{\varepsilon}(\supset \Omega)$ be a cube of side $l+\varepsilon$, and let $\hat{\Phi}_{\lambda}^{(\varepsilon)}$ be the Thomas-Fermi functional obtained by replacing $\Omega$ by $\Omega_{\varepsilon}$ in the definition of $\hat{\Phi}_{\lambda}$. Then, by Theorem 10 ,

$$
F(\lambda, \beta, l+\varepsilon)=\hat{\Phi}_{\lambda}^{(\varepsilon)}\left(\varrho_{\lambda, \varepsilon}, \alpha_{\lambda, \varepsilon}\right),
$$

where $\left(\varrho_{\lambda, \varepsilon}, \alpha_{\lambda, \varepsilon}\right)$ minimises $\hat{\Phi}_{\lambda}^{(\varepsilon)}$. Let

$$
q_{\lambda, \varepsilon}=\int_{\Omega} d^{3} x \varrho_{\lambda, \varepsilon}(x)
$$


and let $\left(\bar{\varrho}_{\lambda, \varepsilon}, \bar{\alpha}_{\lambda, \varepsilon}\right)$ be the restriction of $\left(\varrho_{\lambda, \varepsilon}, \alpha_{\lambda, \varepsilon}\right)$ to $\Omega$. Then it follows from (5.32) and (5.34) that

$$
q_{\lambda, \varepsilon} F(\lambda, \beta, l) \leqq \hat{\Phi}_{\lambda}\left(\bar{\varrho}_{\lambda, \varepsilon}, \bar{\alpha}_{\lambda, \varepsilon}\right),
$$

and hence, by Eqs. (3.5)-(3.7), (5.29). (5.31). (5.33) and (5.35), together with our definitions of $\hat{\Phi}_{\lambda}^{(\varepsilon)}, \bar{\varrho}_{\lambda, \varepsilon}$ and $\bar{\alpha}_{\lambda, \varepsilon}$,

$$
\begin{aligned}
0 \leqq & F(\lambda, \beta, l)-F(\lambda, \beta, l+\varepsilon) \leqq\left(1-q_{\lambda, \varepsilon}\right) F(\lambda, \beta, l) \\
& +\int_{\Omega_{\varepsilon} \backslash \Omega} d^{3} x \hat{\varphi}\left(\varrho_{\lambda, \varepsilon}(x), \alpha_{\lambda, \varepsilon}(x)\right)+\int_{\Omega_{\varepsilon}^{2} \backslash \Omega^{2}} d^{3} x d^{3} y v(x, y) \varrho_{\lambda, \varepsilon}(x) \varrho_{\lambda, \varepsilon}(y) \\
& +\lambda \int_{\Omega_{\varepsilon}^{k} \backslash \Omega^{k}} d^{3} x_{1} \ldots d^{3} x_{k} h\left(x_{1}, \ldots, x_{k}\right) \alpha_{\lambda, \varepsilon}^{(1)}\left(x_{1}\right) \ldots \alpha_{\lambda, \varepsilon}^{(k)}\left(x_{k}\right) .
\end{aligned}
$$

In view of the uniform boundedness conditions given by Lemmas 7-9, it follows easily from (5.35) and (5.36) that

$$
\lim _{\varepsilon \rightarrow 0} F(\lambda, \beta, l+\varepsilon)=F(\lambda, \beta, l)
$$

and hence, by (5.31), we see that the formula (5.30) is valid.

\section{Proof of Lemmas}

Proof of Lemma 5. In view of Eqs. (1.1) and (3.6), it suffices for us to prove that, for us to prove that, for $\varrho_{0} \in \mathbf{R}_{+}$and $\alpha_{0}=\left(\bar{\omega}_{\varrho_{0}}\left(A^{(1)}, \ldots, \bar{\omega}_{\varrho_{0}}\left(A^{(k)}\right)\right)\right.$,

$$
\hat{\varphi}\left(\varrho_{0}, \alpha_{0}\right)=\varphi_{0}\left(\varrho_{0}\right)
$$

and

$$
\hat{\varphi}\left(\varrho_{0}, \alpha_{1}\right)>\varphi_{0}\left(\varrho_{0}\right) \text { for } \alpha_{1} \neq \alpha_{0} .
$$

Let us first prove (6.1). By Eqs. (2.8) and (3.4),

$$
\varphi\left(\varrho_{0}, \alpha_{0}\right)=\varphi_{0}\left(\varrho_{0}\right) \text {. }
$$

Since $\hat{\varphi}$ is the closure of $\varphi$, we can find a sequence $\left\{\left(\varrho_{n}, \alpha_{n}\right)\right.$ in the interior of $\operatorname{Dom} \varphi$, the region where $\varphi$ is finite, such that $\left(\varrho_{n}, \alpha_{n}\right) \rightarrow\left(\varrho_{0}, \alpha_{0}\right)$ and $\varphi\left(\varrho_{n}, \alpha_{n}\right) \rightarrow \hat{\varphi}\left(\varrho_{0}, \alpha_{0}\right)$ as $n \rightarrow \infty\left[13\right.$, p. 52]. Hence, as $\varphi\left(\varrho_{n}, \alpha_{n}\right) \geqq \varphi_{0}\left(\varrho_{n}\right)$, by Eqs. (2.8) and (3.4), it follows that

$$
\hat{\varphi}\left(\varrho_{0}, \alpha_{0}\right) \geqq \limsup _{n \rightarrow \infty} \varphi_{0}\left(\varrho_{n}\right),
$$

and therefore, in view of the continuity of $\varphi_{0}$,

$$
\hat{\varphi}\left(\varrho_{0}, \alpha_{0}\right) \geqq \varphi_{0}\left(\varrho_{0}\right) \text {. }
$$

On the other hand, $\hat{\varphi} \leqq \varphi$, by definition of the closure of a convex function; and therefore, by (6.3) and (6.4), Eq. (6.1) is valid.

We shall prove the inequality (6.2) firstly for the case where $k=1$ and then for arbitrary $k \in \mathbf{Z}_{+}$. For the former case, we start by assuming that, contrary to (6.2), there exists $\alpha_{0}^{\prime} \neq \alpha_{0}$, in $\mathbf{R}$, such that

$$
\hat{\varphi}\left(\varrho_{0}, \alpha_{0}^{\prime}\right) \leqq \varphi_{0}\left(\varrho_{0}\right) \text {. }
$$

For definiteness we shall assume that $\alpha_{0}^{\prime}>\alpha_{0}$ : the case $\alpha_{0}^{\prime}<\alpha_{0}$ can be treated analogously. 
It follows immediately from $(6.5)$ that $\theta_{0}^{\prime}:=\left(\varrho_{0}, \alpha_{0}^{\prime}\right) \in \operatorname{Dom} \hat{\varphi}$. Let $\mathscr{C}$ be the curve $\alpha=a(\varrho):=\bar{\omega}_{\varrho}(A)$, which is continuous because of the $w^{*}$-continuity of $\bar{\omega}_{\varrho}$ in $\varrho$. Since $\hat{\varphi}(\varrho, a(\varrho))=\varphi_{0}(\varrho)\left[\right.$ cf. (6.1)] it follows that $\mathscr{C}$ also lies in Dom $\hat{\varphi}$. Let $\varrho_{1}, \varrho_{2}$ be two positive numbers such that $\varrho_{1}<\varrho_{0}<\varrho_{2}$, and let $\theta_{i}:=\left(\varrho_{i}, a\left(\varrho_{i}\right)\right)$ for $i=1,2$. We define $K$ to be the interior of the domain bounded by the curve $\mathscr{C}$ and the lines connecting $\theta_{0}^{\prime}$ to $\theta_{1}$ and $\theta_{2}$ : thus, as we are taking $\alpha_{0}^{\prime}$ to be greater than $\alpha_{0}$,

$$
K=\left\{(\varrho, \alpha) \mid \alpha>a(\varrho) ; \alpha<a\left(\varrho_{i}\right)+\frac{\alpha_{0}^{\prime}-a\left(\varrho_{i}\right)}{\varrho_{0}-\varrho_{i}}\left(\varrho-\varrho_{i}\right) \text { for } i=1,2\right\} .
$$

Since $\theta_{0}^{\prime}$ and $\mathscr{C}$ lie in $\operatorname{Dom} \hat{\varphi}$, it follows from the convexity of $\hat{\varphi}$ that $K \subset \operatorname{Int} \operatorname{Dom} \hat{\varphi}$; and therefore $\varphi$ and $\hat{\varphi}$ coincide in $K$ [13, Theorem 7.4]. Hence as $\left(\varrho_{0}, \frac{1}{2}\left(\alpha_{0}+\alpha_{0}^{\prime}\right)\right) \in K$, it follows that

$$
\hat{\varphi}\left(\varrho_{0}, \frac{1}{2}\left(\alpha_{0}+\alpha_{0}^{\prime}\right)\right)=\varphi\left(\varrho_{0}, \frac{1}{2}\left(\alpha_{0}+\alpha_{0}^{\prime}\right) .\right.
$$

Further, as $\hat{\varphi}$ is jointly convex in its arguments,

$$
\begin{aligned}
\hat{\varphi}\left(\varrho_{0}, \frac{1}{2}\left(\alpha_{0}+\alpha_{0}^{\prime}\right)\right) & \leqq \frac{1}{2} \hat{\varphi}\left(\varrho_{0}, \alpha_{0}\right)+\frac{1}{2} \hat{\varphi}\left(\varrho_{0}, \alpha_{0}^{\prime}\right) \\
& \leqq \varphi\left(\varrho_{0}, \alpha_{0}\right), \quad \text { by }(6.3) \text { and }(6.4) ;
\end{aligned}
$$

and consequently, by (6.6),

$$
\varphi\left(\varrho_{0}, \frac{1}{2}\left(\alpha_{0}+\alpha_{0}^{\prime}\right)\right) \leqq \varphi\left(\varrho_{0}, \alpha_{0}\right) .
$$

However, as the free energy density functional for the ideal Fermi gas at given density is minimised at the unique state $\bar{\omega}_{\varrho}$ (cf. Appendix 2, Theorem A2.1), it follows from Eq. (3.10) that (6.7) cannot be valid when $\alpha_{0}^{\prime} \neq \alpha_{0}$. In other words, we have established that the assumption of (6.5) cannot be valid with $\alpha_{0}^{\prime} \neq \alpha_{0}$, and thereby proved the inequality (6.2) for the case when $k=1$.

In the case where $k>1$, we define $\varphi_{i}: \mathbf{R}_{+} \times \mathbf{R} \rightarrow \mathbf{R}_{\cup}\{\infty\}$, for $i=1, \ldots, k$, by the formula

$$
\varphi_{i}\left(\varrho, \alpha^{(i)}\right)=\left\{\begin{array}{l}
\inf \left\{f(\omega) \mid n(\omega)=\varrho ; \omega\left(A^{(i)}\right)=\alpha^{(i)}\right\} \quad \text { if } \quad \exists \omega \in \mathscr{S}(\mathscr{A}) \\
n(\omega)=\varrho, \omega\left(A^{(i)}\right)=\alpha^{(i)} ; \text { and }=\infty \text { otherwise }
\end{array}\right.
$$

Hence by Eqs. (3.4) and (6.8)

$$
\varphi(\varrho, \alpha) \geqq \varphi_{i}\left(\varrho, \alpha^{(i)}\right) ; \alpha=\left(\alpha^{(1)}, \ldots, \alpha^{(k)}\right) .
$$

In order to reduce our proof of (6.2) to the one we have already carried out for $k=1$, it suffices to show that

$$
\hat{\varphi}(\varrho, \alpha) \geqq \hat{\varphi}_{i}\left(\varrho, \alpha^{(i)}\right),
$$

where $\hat{\varphi}_{i}$ is the closure of $\varphi_{i}$. This we now do as follows. In the non-trivial case where the L.H.S. of (6.10) is finite, we may choose a sequence $\left(\varrho_{n}, \alpha_{n}\right) \in \operatorname{Dom} \varphi$ such that $\left(\varrho_{n}, \alpha_{n}\right) \rightarrow(\varrho, \alpha)$ and $\varphi\left(\varrho_{n}, \alpha_{n}\right) \rightarrow \hat{\varphi}(\varrho, \alpha)$ as $n \rightarrow \infty$. Hence

$$
\begin{aligned}
\hat{\varphi}(\varrho, \alpha) & \left.=\lim _{n \rightarrow \infty} \varphi\left(\varrho_{n}, \alpha_{n}\right) \geqq \limsup _{n \rightarrow \infty} \varphi_{i}\left(\varrho_{n}, \alpha_{n}^{(i)}\right) \quad \text { (by }(6.9)\right) \\
& \geqq \limsup _{n \rightarrow \infty} \hat{\varphi}_{i}\left(\varrho_{n}, \alpha_{n}^{(i)}\right) \quad\left(\text { as } \varphi_{i} \geqq \hat{\varphi}_{i}\right) \\
& \geqq \hat{\varphi}_{i}\left(\varrho, \alpha^{(i)}\right) \quad\left(\text { by lower semicontinuity of } \hat{\varphi}_{i}\right) .
\end{aligned}
$$


Proof of Lemma 6. It follows from the lower semicontinuity of $\hat{\varphi}$ that $\hat{\varphi}^{-1}(-\infty, B]$ is closed, and hence that $\Theta^{(B)}$ is a closed subset of $\Theta$. Let $(\varrho, \alpha) \in \Theta^{(B)}$. Then by Eqs. (6.1), (6.2) and the definition of $\Theta^{(B)}$,

$$
\varphi_{0}(\varrho(x)) \leqq \hat{\varphi}(\varrho(x), \alpha(x)) \leqq B \quad \text { for } \quad x \text { a.e. in } \Omega .
$$

Since the function $\varphi_{0}: \mathbf{R}_{+} \rightarrow \mathbf{R}$ is bounded on the compacts, continuous, lowerbounded and tending to $\infty$ at $\infty$, it follows from (6.11) that $\exists \bar{\varrho}_{B} \in \mathbf{R}_{+}$such that $\varrho(x) \leqq B$ for $x$ a.e. in $\Omega$, i.e. $\|\varrho\|_{\infty} \leqq \varrho_{B}$. It also follows from (6.11) that, for $x$ a.e. in $\Omega$, $(\varrho(x), \alpha(x)) \in \operatorname{Dom} \hat{\varphi}$ and hence belongs to the closure of Dom $\varphi$. Therefore, by Eq. (3.4), $\left\|\alpha^{(i)}\right\|_{\infty} \leqq\left\|A^{(i)}\right\|$ for $i=1, \ldots, k$. Thus, we have proved that $\Theta^{(B)}$ is a closed subset of the compact metrisable space

$$
\Theta_{1}^{(B)}:=\left\{\theta=\left(\varrho ; \alpha^{(1)}, \ldots, \alpha^{(x)}\right) \in \Theta \mid\|\varrho\|_{\infty} \leqq \bar{\varrho}_{B} ;\left\|\alpha^{(i)}\right\|_{\infty} \leqq\left\|A^{(i)}\right\| \quad \text { for } \quad i=1, \ldots, k\right\},
$$

and is therefore itself compact and metrisable.

Proof of Lemma 7. $\hat{\Phi}_{\lambda}$ is defined by Eqs. (3.5)-(3.7). It follows from the uniform boundedness of the elements of $\Theta^{(B)}$ (cf. Lemma 7), together with the fact that $v \in L_{1}\left(\Omega^{2}\right)$ and $h$ is bounded, that the contributions to $\hat{\Phi}_{\lambda}$ given by $\lambda \Psi$ and by the last term on the R.H.S. of (3.6) are both continuous. Hence, in order to establish the lower semicontinuity of $\hat{\Phi}_{\lambda}$, and likewise of $\hat{\Phi}_{\mu \lambda}$, it suffices for us to prove that the mapping $\theta\left(\in \Theta^{(B)}\right) \rightarrow \int_{\Omega} d^{3} x \hat{\varphi}(\theta(x))$ possesses this property.

For this purpose, we resolve $\Omega$ into cells, $C_{1}, \ldots, C_{g}$; and, for $\theta \in \Theta^{(B)}$, we define $\theta_{g}$ to be the element of $\Theta^{(B)}$ obtained by replacing $\theta$ in each cell $C_{r}$ by its mean value, $\bar{\theta}_{r}$, over $C_{r}$. We then define

$$
G(\theta)=\int_{\Omega} d^{3} x \hat{\varphi}(\theta(x))
$$

and

$$
G_{g}(\theta)=\int_{\Omega} d^{3} x \hat{\varphi}\left(\theta_{g}(x)\right) \equiv \sum_{r=1}^{g} \hat{\varphi}\left(\bar{\theta}_{r}\right)\left|C_{r}\right| .
$$

Since the elements of $\Theta^{(B)}$ are uniformly bounded (cf. Lemma 7), it follows that the mapping $\theta \rightarrow \bar{\theta}_{r}$ is continuous. Hence, by (6.13), as $\hat{\varphi}$ is lower semicontinuous, so too is $G_{g}$.

By Lusin's theorem, $\theta_{g}(x)$ converges pointwise to $\theta(x)$, except on a set of arbitrarily small measure, as $g \rightarrow \infty$. Hence, as $\hat{\varphi}$ is bounded and lower semicontinuous, it follows from Eqs. (6.12), (6.13), together with Fatou's lemma, that

$$
\liminf _{g \rightarrow \infty} G_{g}(\theta)=G(\theta)
$$

On the other hand, as $\hat{\varphi}$ is convex, we see from $(6.12),(6.13)$ that $G_{g}(\theta) \leqq G(\theta)$. Therefore, Eq. (6.14) implies that $G$ is the supremum of a family $\left\{G_{g}\right\}$ of lower semicontinuous functions on $\Theta^{(B)}$ and is therefore itself lower semicontinuous.

Proof of Lemma 8. Our method here is an extension of that used in Ref. [5] for the proof of the uniform boundedness of the density.

We shall employ the following notation: $\sigma:=(\mu, g, \lambda, \beta, l)$, with $|\lambda|<\lambda_{0}$, $\beta \in\left(\beta_{1}, \beta_{2}\right), l \in\left(l_{1}, l_{2}\right) ;\left(\varrho_{\sigma}, \bar{\alpha}_{\sigma}\right)$ denotes an element of $\Theta_{g}$ at which $\hat{\Phi}_{\mu g \lambda}$ is minimised; 
$\left(\varrho_{\sigma r}, \bar{\alpha}_{\sigma r}\right)$ denotes the value of $\left(\varrho_{\sigma}, \bar{\alpha}_{\sigma}\right)$ in the cell $C_{r}$; and $v_{r s}, h_{r_{1}, \ldots, r_{k}}$ are as defined by Eqs. (5.11), (5.12). Thus, the increment $\Delta_{t}$ in the value of $\hat{\Phi}_{\mu g \lambda}$ when its argument is changed from $\left(\bar{\varrho}_{\sigma}, \bar{\alpha}_{\sigma}\right)$ due to increments $\left|C_{r_{1}}\right|^{-1} t$ and $-\left|C_{r_{2}}\right|^{-1} t$ in the densities in $C_{r_{1}}, C_{r_{2}}$, respectively, is non-negative. Hence, it follows from the definition of $\hat{\Phi}_{\mu g \lambda}$, in Sect. 4(iv), together with the convexity of $\hat{\varphi}$ and Eqs. (3.5)-(3.7), (5.11), (5.12),

that the inequality $\lim _{t \rightarrow+0} \Delta_{t} / t \geqq 0$ yields the following result:

$$
\begin{aligned}
& \hat{\varphi}_{\varrho}^{(+)}\left(\bar{\varrho}_{\sigma r_{1}}, \bar{\alpha}_{\sigma r_{1}}\right)+\sum_{s} v_{r_{1} s} \bar{\varrho}_{\sigma s}\left|C_{s}\right| \\
& \quad \geqq \hat{\varphi}_{\varrho}^{(-)}\left(\bar{\varrho}_{\sigma r_{2}}, \bar{\alpha}_{\sigma r_{2}}\right)+\sum_{s} v_{r_{2} s} \bar{\varrho}_{\sigma s}\left|C_{s}\right|,
\end{aligned}
$$

where $\hat{\varphi}_{\varrho}^{( \pm)}$denote the right and left derivatives, respectively, of $\hat{\varphi}$ w.r.t. $\varrho$. Since this result is valid for all pairs of cells $C_{r_{1}}, C_{r_{2}}$, it follows that

$$
\begin{aligned}
& \min _{r}\left[\hat{\varphi}_{\varrho}^{(+)}\left(\bar{\varrho}_{\sigma r}, \bar{\alpha}_{\sigma r}\right)+\sum_{s} v_{r s} \bar{\varrho}_{\sigma s}\left|C_{s}\right|\right] \\
& \geqq \max _{r}\left[\hat{\varphi}_{\varrho}^{(-)}\left(\varrho_{\sigma r}, \bar{\alpha}_{\sigma r}\right)+\sum_{s} v_{r s} \bar{\varrho}_{\sigma s}\left|C_{s}\right|\right],
\end{aligned}
$$

and hence, there exists a quantity $\hat{\eta}_{\sigma}$, independent of $r$, such that

$$
\hat{\varphi}_{\varrho}^{(+)}\left(\bar{\varrho}_{\sigma r}, \bar{\alpha}_{\sigma r}\right) \geqq \bar{\eta}_{\sigma r} \geqq \hat{\varphi}_{\varrho}^{(-)}\left(\bar{\varrho}_{\sigma r}, \bar{\alpha}_{\varrho r}\right), \quad \text { for } \quad r=1, \ldots, g,
$$

where

$$
\bar{\eta}_{\sigma r}:=\hat{\eta}_{\sigma}-\sum_{s} v_{r s} \bar{\varrho}_{\sigma s} .
$$

Likewise, by considering the increments in $\hat{\Phi}_{\mu g \lambda}$. when $\alpha$ is changed from $\bar{\alpha}_{\sigma r}$ to $\bar{\alpha}_{\sigma r} \pm t$ in the cell $C_{r}$ only, and leaving $\varrho$ unchanged at $\varrho_{\sigma}$, we find that

$$
\hat{\varphi}_{i}^{(+)}\left(\bar{\varrho}_{\sigma r}, \bar{\alpha}_{\sigma r}\right) \geqq \bar{y}_{\sigma r}^{(i)} \geqq \hat{\varphi}_{i}^{(-)}\left(\bar{\varrho}_{\sigma r}, \bar{\alpha}_{\sigma r}\right),
$$

where $\hat{\varphi}_{i}{ }^{( \pm)}$denote the right and left derivatives, respectively, of $\hat{\varphi}$ w.r.t. $\alpha^{(i)}$, and

$$
\bar{y}_{\sigma r}^{(i)}=\lambda \sum_{r_{1}, \ldots, r_{k}} \delta_{r r_{i}} h_{r_{1} \ldots r_{k}} \prod_{j \neq i} \bar{\alpha}_{\sigma r_{j}}^{(j)}\left|C_{r_{j}}\right| \text {. }
$$

Since $\varphi_{0}$ and therefore $\hat{\varphi}$ is lower-bounded [cf. Eq. (6.2)], and since $\left(\bar{\varrho}_{\sigma}, \bar{\alpha}_{\sigma}\right)$ minimises $\hat{\Phi}_{\mu g \lambda}$, it follows that $\left(\bar{\varrho}_{\sigma r}, \bar{\alpha}_{\sigma r}\right) \in \operatorname{Dom} \hat{\varphi} \subset C l(\operatorname{Dom} \varphi)$, and consequently, by (3.4), $\left|\alpha_{\sigma r}^{(i)}\right| \leqq\left\|A^{(i)}\right\|$. Hence, in view of the boundedness of $h$ and $\lambda$, it follows from (6.19) that one can find a finite constant $b$, independent of $\sigma, i$ and $r$, such that

$$
\left|\bar{y}_{\sigma r}^{(i)}\right|<b \text {. }
$$

Let $\psi, \psi_{0}$ be the real-valued functions on $\mathbf{R}_{+} \times \mathbf{R}^{k}$ and $\mathbf{R}^{k}$, respectively given by the equations

$$
\psi(\eta, y)=\inf _{\varrho, \alpha}[\hat{\varphi}(\varrho, \alpha)-\eta \varrho-y . \alpha] ; \quad y . \alpha=\sum_{i=1}^{k} y^{(i)} \alpha^{(i)}
$$

and

$$
\psi_{0}(\eta)=\inf _{\varrho}\left[\varphi_{0}(\varrho)-\eta \varrho\right]
$$


It follows easily from these definitions that $\psi$ is jointly concave in its arguments; and that $\psi_{0}$, which is the Gibbs free energy for the ideal Fermi gas at chemical potential $\eta$, is a concave function. Further,

$$
\psi_{0}(\eta)=\psi(\eta, 0)
$$

since, by (6.1) and (6.2), $\varphi_{0}(\varrho)=\inf _{\alpha} \hat{\varphi}(\varrho, \alpha)$; and, as the infinum in (6.21) is unaffected by the restriction that $(\alpha, \varrho) \in \operatorname{Dom} \hat{\varphi}$, and thus that $\left|\alpha^{(i)}\right| \leqq\left\|A^{(i)}\right\|$, it follows from (6.21)-(6.23) that

$$
\left|\psi(\eta, y)-\psi_{0}(\eta)\right| \leqq \sum_{i=1}^{k}\left|y^{(i)}\right|\left\|A^{(i)}\right\| .
$$

In view of (6.18) and (6.19), it follows from the convexity of $\varphi$ that, when $(\eta, y)$ $=\left(\bar{\eta}_{\sigma r}, \bar{y}_{\sigma r}\right)$, the infinum on the R.H.S. of $(6.21)$ is attained for $(\varrho, \alpha)=\left(\bar{\varrho}_{\sigma r}, \bar{\alpha}_{\sigma r}\right)$; and that

$$
\psi(\eta, y)-\psi\left(\bar{\eta}_{\sigma r}, \bar{y}_{\sigma r}\right) \leqq-\left(\eta-\bar{\eta}_{\sigma r}\right) \bar{\varrho}_{\sigma r}-\left(y-\bar{y}_{\sigma r}\right), \bar{\alpha}_{\sigma r} .
$$

Hence, as $\psi$ is jointly concave in its arguments, $\left(-\bar{\varrho}_{\sigma r},-\bar{\alpha}_{\sigma r}\right)$ is tangent to $\psi$ at $\left(\bar{\eta}_{\sigma r}, \bar{y}_{\sigma r}\right)$, and therefore

$$
-\psi_{\eta}^{(-)}\left(\bar{\eta}_{\sigma r}, \bar{y}_{\sigma r}\right) \leqq \bar{\varrho}_{\sigma r} \leqq-\psi^{(+)}\left(\bar{\eta}_{\sigma r}, \bar{y}_{\sigma r}\right),
$$

where $\psi_{\eta}^{( \pm)}$are the right and left derivatives, respectively, of $\psi$ w.r.t. $\eta$.

Now, by (6.20) and (6.24), one can find a finite constant $c$, independent of $\sigma$ and $r$, such that

$$
\left|\psi\left(\eta, \bar{y}_{\sigma r}\right)-\psi_{0}(\eta)\right|<c \forall \eta \in \mathbf{R} .
$$

Thus, choosing $p$ to be some positive constant,

$$
\frac{\psi\left(\eta+p, \bar{y}_{\sigma r}\right)-\psi\left(\eta, \bar{y}_{\sigma r}\right)}{p}-\frac{\psi_{0}(\eta+p)-\psi_{0}(\eta)}{p}>-\frac{2 c}{p} ;
$$

and hence, in view of the convexity of $\psi$ and $\psi_{0}$, as well as the differentiability of $\psi_{0}$,

$$
\psi_{\eta}^{(+)}\left(\eta, \bar{y}_{\sigma r}\right)>\psi_{0}^{\prime}(\eta+p)-2 c / p,
$$

where $\psi_{0}^{\prime}$ is the derivative of $\psi_{0}$. Similarly,

$$
\psi_{\eta}^{(-)}\left(\eta, \bar{y}_{\sigma r}\right)<\psi_{0}^{\prime}(\eta-p)+2 c / p .
$$

Therefore, by (6.25)-(6.27),

$$
\bar{\varrho}_{\sigma r}<-\psi_{0}^{\prime}(\eta+p)+2 c / p
$$

and

$$
\bar{\varrho}_{\sigma r}>-\psi_{0}^{\prime}(\eta-p)-2 c / p .
$$

It may now be seen that one can adapt the argument of [5, Sect. 4] to infer from Eqs. (6.17), (6.28), (6.29) and the behaviour of $\psi_{0}^{\prime}(\eta)\left(\sim-\eta^{3 / 2}\right)$ for large $\eta$, that $\bar{\varrho}_{\sigma r}$ is uniformly bounded w.r.t. $\sigma$ and $r$. Specifically, one can do this by using the 
arguments of that article to show first that (6.17) and (6.29) imply that $\hat{\eta}_{\sigma}$ has a finite upper bound; and then inferring from this result and Eqs. (6.17), (6.28) that $\bar{\varrho}_{\sigma r}$ is uniformly bounded.

In order to establish a similar result for $\hat{\varphi}\left(\bar{\varrho}_{\sigma r}, \bar{\alpha}_{\sigma r}\right)$, we note that $\hat{\Phi}_{\mu g \lambda}$ cannot be decreased if its argument is altered from $\left(\bar{\varrho}_{\sigma}, \bar{\alpha}_{\sigma}\right)$ by changing $\bar{\alpha}_{\sigma r}$ to $\bar{\omega}_{\varrho_{\sigma r}}(A)$. Hence, it follows from the definition of $\hat{\Phi}_{\mu g \lambda}$, as given in Sect.4(iv) together with Eqs. (3.5)-(3.7), that, in view of (6.1),

$$
\varphi_{0}\left(\bar{\varrho}_{\sigma r}\right) \geqq \hat{\varphi}\left(\bar{\varrho}_{\sigma r}, \bar{\alpha}_{\sigma r}\right)+\bar{y}_{\sigma r} \cdot\left(\bar{\alpha}_{\sigma r}-\bar{\omega}_{\bar{\varrho}_{\sigma r}}(A)\right) .
$$

Thus, in view of the uniform boundedness of $\bar{y}_{\sigma r}[\mathrm{cf} .(6.20)]$ and $\bar{\alpha}_{\sigma r}$, we can find a constant $d$, independent of $\sigma$ and $r$, such that $\hat{\varphi}\left(\varrho_{\sigma r}, \bar{\alpha}_{\sigma r}\right)<\varphi_{0}\left(\varrho_{\sigma r}\right)+d$; and therefore, as $\varphi_{0}$ is bounded on the compacts and $\varrho_{\sigma r}$ is uniformly bounded, it follows that $\hat{\varphi}\left(\bar{\varrho}_{\sigma r}, \bar{\alpha}_{\sigma r}\right)$ is uniformly upper bounded.

\section{Appendix 1: Mean Field Theory}

In order to avoid inessential notational complications, we confine our derivation of the formula (5.20) to the case where $g=2$ and $h_{r_{1} r_{2}}=0$ except when $r_{1}=1, r_{2}=2$. The full proof of (5.20) for the general case can be carried out analogously.

Thus, we replace the formula $(5.15)$ by the following simpler one:

$$
\tilde{H}_{N, \varrho}=\tilde{T}_{1} \otimes \tilde{I}_{2}+\tilde{I}_{1} \otimes \tilde{T}_{2}+N \tilde{A}_{1}^{(1)} \otimes \tilde{A}_{2}^{(2)}+N v \varrho_{1} \varrho_{2},
$$

where $v=v_{12},\left|C_{1}\right|=\left|C_{2}\right|=1$ and $\lambda$ is absorbed into $\tilde{A}_{1}^{(1)} \otimes \tilde{A}_{2}^{(2)}$. Equation (4.9) can now be expressed in the form

$$
\bar{F}_{N}=\min \left\{N^{-1} \tilde{\operatorname{T}} \mathrm{r}\left(\tilde{\sigma} \ln \tilde{\sigma}+\tilde{\sigma} \tilde{H}_{N, \varrho}\right) \mid \varrho \in P_{N} ; \tilde{\sigma} \in D_{N, \varrho}\right\},
$$

where $P_{N}$ is as defined in Sect. 4(iv), $D_{N, \varrho}$ denotes the set of density matrices in $\mathscr{H}_{N, \varrho}, \beta$ is taken to be equal to 1 and the parameters $\mu, l$ are omitted. We define $\bar{F}_{N}^{(0)}$ to be the corresponding quantity when the density matrices are restricted to those without intercellular correlations, i.e.

$$
\bar{F}_{N}^{(0)}=\min \left\{N^{-1} \tilde{\operatorname{Tr}}\left(\tilde{\sigma} \ln \tilde{\sigma}+\tilde{\sigma} \tilde{H}_{N, \varrho}\right) \mid \varrho \in P_{N} ; \tilde{\sigma}=\tilde{\sigma}_{1} \otimes \tilde{\sigma}_{2} \in D_{N, \varrho}\right\}
$$

the trace in this expression attaining its infinum, as it corresponds to a lower semicontinuous function on a compact set (cf. [12]). We shall now establish (5.20), for the model treated here, in two stages. In the first of these, we shall prove that

$$
\lim _{N \rightarrow \infty}\left(\bar{F}_{N}-\bar{F}_{N}^{(0)}\right)=0 ;
$$

and in the second we shall show that

$$
\lim _{N \rightarrow \infty} \bar{F}_{N}^{(0)}=\min \left\{\hat{\Phi}\left(\varrho_{1}, \varrho_{2} ; \alpha_{1}, \alpha_{2}\right) \mid \varrho_{1}, \varrho_{2} \in \mathbf{R}_{+} ; \varrho_{1}+\varrho_{2}=1 ; \alpha_{1}, \alpha_{2} \in \mathbf{R}\right\}
$$

with

$$
\hat{\Phi}\left(\varrho_{1}, \varrho_{2} ; \alpha_{1}, \alpha_{2}\right)=\hat{\varphi}\left(\varrho_{1}, \alpha_{1}\right)+\hat{\varphi}\left(\varrho_{2}, a_{2}\right)+\alpha_{1} \alpha_{2}+v \varrho_{1} \varrho_{2} .
$$

Equations (A 1.4)-(A1.6) imply the desired result, corresponding to (5.22). 
Stage 1. It follows immediately from (A1.2) and (A1.3) that

$$
\bar{F}_{N} \leqq \bar{F}_{N}^{(0)} \text {. }
$$

In order to obtain an upper bound for $\bar{F}_{N}^{(0)}-\bar{F}_{N}$, we first note that the values of $\tilde{\sigma}$, $\varrho$, for which the minimum in (A1.2) is achieved, satisfy the relation

$$
\tilde{\sigma}=\exp \left(-\tilde{H}_{N, \varrho}\right) / \tilde{\operatorname{Tr}} \text { (idem) }
$$

Let

$$
\tilde{\sigma}^{\prime}=\tilde{\sigma}_{1} \otimes \tilde{\sigma}_{2}
$$

where

$$
\tilde{\sigma}_{1}=\tilde{\operatorname{Tr}}_{2} \tilde{\sigma} ; \quad \tilde{\sigma}_{2}=\tilde{\operatorname{Tr}}_{1} \tilde{\sigma}
$$

and $\operatorname{Tr}_{i}$ is the partial trace over $\tilde{\mathscr{H}}_{i}\left(:=\mathscr{H}_{N \ell_{i}}\left(\tilde{C}_{i}\right)\right)$. It follows from (A1.1)-(A1.3) that

$$
\begin{aligned}
\bar{F}_{N}^{(0)} & \leqq N^{-1} \tilde{\operatorname{Tr}}\left(\tilde{\sigma}^{\prime} \ln \tilde{\sigma}^{\prime}+\tilde{\sigma}^{\prime} \tilde{H}_{N, \varrho}\right) \\
& =N^{-1}\left[\sum_{i=1}^{2} \tilde{\operatorname{Tr}} \tilde{r}_{i}\left(\tilde{\sigma}_{i} \ln \tilde{\sigma}_{i}\right)+\tilde{\operatorname{Tr}}\left(\tilde{\sigma}^{\prime} \tilde{H}_{N, \varrho}\right)\right] \\
& \leqq N^{-1} \tilde{\operatorname{Tr}}\left(\tilde{\sigma} \ln \tilde{\sigma}+\tilde{\sigma}^{\prime} \tilde{H}_{N, \varrho}\right) \quad \text { (subadditivity of entropy) }
\end{aligned}
$$

i.e.

$$
\bar{F}_{N}^{(0)} \leqq \bar{F}_{N}+N^{-1} \tilde{\operatorname{Tr}}\left(\left(\tilde{\sigma}^{\prime}-\tilde{\sigma}\right) \tilde{H}_{N, \varrho}\right) .
$$

In order to utilise the techniques of [8], we introduce the "perturbed Hamiltonian"

$$
\tilde{H}_{N, \varrho}(x):=\tilde{H}_{N, \varrho}+N x_{1} \tilde{A}_{1}^{(1)} \otimes \tilde{I}_{2}+N x_{2} \tilde{I}_{1} \otimes \tilde{A}_{2}^{(2)} ; x=\left(x_{1}, x_{2}\right) \in \mathbf{R}^{2} ;
$$

and we define $\bar{F}_{N}(x), \bar{F}_{N}^{(0)}(x), \tilde{\sigma}(x), \tilde{\sigma}^{\prime}(x), \sigma_{i}(x)$ to be the quantities obtained on replacement of $\tilde{H}_{N, \varrho}$ by $H_{N, \varrho}(x)$ in the formulae for $\bar{F}_{N}, \bar{F}_{N}^{(0)}, \tilde{\sigma}, \tilde{\sigma}^{\prime}, \tilde{\sigma}_{i}$, respectively. Hence, by (A 1.1), (A 1.7) and (A 1.9)-(A 1.12),

$$
\begin{aligned}
0 & \leqq \bar{F}_{N}^{(0)}(x)-\bar{F}_{N}(x) \leqq \tilde{T} r\left(\left(\tilde{\sigma}^{\prime}(x)-\tilde{\sigma}(x)\right)\left(\tilde{A}_{1}^{(1)} \otimes \tilde{A}_{2}^{(2)}\right)\right) \\
& =-\frac{1}{2}\left\langle\left(\tilde{A}_{1}^{(1)}-\left\langle\tilde{A}_{1}^{(1)}\right\rangle_{\tilde{\sigma}_{1}(x)} \otimes \tilde{A}_{2}^{(2)}\right\rangle_{\tilde{\sigma}(x)}-\frac{1}{2}\left\langle\tilde{A}_{1}^{(1)} \otimes\left(\tilde{A}_{2}^{(2)}-\left\langle\tilde{A}_{2}^{(2)}\right\rangle_{\tilde{\sigma}_{2}(x)}\right)\right\rangle_{\tilde{\sigma}(x)} .\right.
\end{aligned}
$$

Therefore, as Eq. (5.16) implies that $\left\|\tilde{A}_{i}^{(i)}\right\| \leqq\left\|A^{(i)}\right\|$, we see that

$$
0 \leqq \bar{F}_{N}^{(0)}(x)-\bar{F}_{N}(x) \leqq c \sum_{i=1,2}\left\langle\left(\tilde{A}_{i}^{(i)}-\left\langle\tilde{A}_{i}^{(i)}\right\rangle_{\tilde{\sigma}_{i}(x)}\right)^{2}\right\rangle_{\tilde{\sigma}_{l}(x)}^{1 / 2},
$$

where $c$ is a constant, chosen to exceed $\frac{1}{2}\left(\left\|A^{(1)}\right\|+\left\|A^{(2)}\right\|\right)$. In view of Eq. (5.16), one can easily find a subset $\mathscr{A}_{0}$ of $\mathscr{A}_{2}$ that is dense in $\mathscr{A}$, such that $\left\{\tilde{A}_{i}^{(i)}\right\}$ satisfy the conditions corresponding to [8; Eq. 7] for all $A^{(1)}, A^{(2)} \in \mathscr{A}_{0}$. Consequently for such $A^{(1)}, A^{(2)}$, Eq. (A1.12) is amenable to the same treatment as a similar formula in [8], and may thus be shown to imply that

$$
0 \leqq \bar{F}_{N}^{(0)}(x)-\bar{F}_{N}(x) \leqq \gamma_{1} N^{-1 / 2}\left(-\Delta \bar{F}_{N}(x)\right)^{1 / 2}+\gamma_{2} N^{-2 / 3}\left(-\Delta \bar{F}_{N}(x)\right)^{2 / 3},
$$

where $\gamma_{1}, \gamma_{2}$ are finite positive constants, and $\Delta$ is the two-dimensional Laplacian; and thence that Eq. (A1.4) is valid. This result is extended by continuity to arbitrary $A^{(1)}, A^{(2)} \in \mathscr{A}_{L}$. 
Stage 2. Let $\tilde{\sigma}_{1} \otimes \tilde{\sigma}_{2}$ and $\bar{\varrho}^{(N)}$ correspond to the values of the density matrix and particle distribution, respectively, at which the Trace in (A1.3) is minimised. Then, if $\tilde{\sigma}_{1}^{\prime}$ is any other density matrix in $\tilde{\mathscr{H}}_{i}$, the replacement of $\tilde{\sigma}_{i}$ by $\sigma_{i}^{\prime}$ cannot decrease the value of that Trace. Hence, using (A1.1)

$$
\tilde{\operatorname{Tr}}_{i}\left(\tilde{\sigma}_{i} \ln \tilde{\sigma}_{i}+\tilde{\sigma}_{i}\left(\tilde{T}_{i}+\bar{y}_{i}^{(N)} \tilde{A}_{i}^{(i)}\right)\right) \leqq \tilde{\operatorname{Tr}}_{i}\left(\tilde{\sigma}_{i}^{\prime} \ln \tilde{\sigma}_{i}^{\prime}+\tilde{\sigma}_{i}^{\prime}\left(\tilde{T}_{i}+\bar{y}_{i}^{(N)} \tilde{A}_{i}^{(i)}\right)\right),
$$

where

$$
\bar{y}_{1}^{(N)}=\tilde{\mathrm{T}}_{2}\left(\tilde{\sigma}_{2} \tilde{A}_{2}^{(2)}\right) ; \quad \bar{y}_{2}^{(N)}=\tilde{\mathrm{T}}_{1}\left(\tilde{\sigma}_{1} \tilde{A}_{1}^{(1)}\right) .
$$

(A 1.14) constitutes a variational principle, from which it follows that

$$
\tilde{\sigma}_{i}=\exp -\left(\tilde{T}_{i}+\bar{y}_{i}^{(N)} \tilde{A}_{i}^{(i)}\right) / \tilde{T}_{i}(\mathrm{idem}) .
$$

Thus, by (A1.1), (A1.3) and (A1.16),

$$
\bar{F}_{N}^{(0)}=\sum_{i=1,2}\left(\psi_{N}\left(\bar{\varrho}_{i}^{(N)}, \bar{y}_{i}^{(N)}\right)-\bar{\alpha}_{i}^{(N)} \bar{y}_{i}^{(N)}\right)+\bar{\alpha}_{1}^{(N)} \bar{\alpha}_{2}^{(N)}+v \bar{\varrho}_{1}^{(N)} \bar{\varrho}_{i}^{(N)}
$$

where

$$
\psi_{N}\left(\bar{\varrho}_{i}^{(N)}, y_{i}\right)=-\ln \tilde{T}_{i} \exp -\left(\tilde{T}_{i}+y_{i} \tilde{A}_{i}^{(i)}\right)
$$

and

$$
\bar{\alpha}_{i}^{(N)}=\tilde{\operatorname{T}}_{i}\left(\tilde{\sigma}_{i} \tilde{A}_{i}^{(i)}\right) .
$$

It follows from these last two equations that $\psi_{N}$ is concave in $y_{i}$ and that

$$
\psi_{N}\left(\bar{\varrho}_{i}^{(N)}, y_{i}\right)-\psi_{N}\left(\bar{\varrho}_{i}^{(N)}, \bar{y}_{i}^{(N)}\right) \leqq\left(y_{i}-\bar{y}_{i}^{(N)}\right) \bar{\alpha}_{i}^{(N)}
$$

By (5.16), (A 1.20) and (A 1.21), the sequences $\left\{\bar{\varrho}_{i}^{(N)}\right\},\left\{\bar{\alpha}_{i}^{(N)}\right\}$ and $\left\{\bar{y}_{i}^{(N)}\right\}$ are uniformly bounded, as $N$ runs through $\mathbf{Z}_{+}$, and therefore have accumulation points $\bar{\varrho}_{i}, \bar{\alpha}_{i}$ and $\bar{y}_{i}$, respectively. Correspondingly (cf. [10; Proposition 3.5.10]), $\left\{\psi_{N}\left(\bar{\varrho}_{i}^{(N)}, \bar{y}_{i}^{(N)}\right)\right\}$ has an accumulation point $\psi\left(\bar{\varrho}_{i}, \bar{y}_{i}\right)$, where $\psi$ is the thermodynamic potential defined by the formula

$$
\psi\left(\bar{\varrho}_{i}, y_{i}\right)=\lim _{N \rightarrow \infty} \psi_{N}\left(\bar{\varrho}_{i}^{(N)}, y_{i}\right) ;
$$

or equivalently [12],

$$
\psi\left(\bar{\varrho}_{i}, y_{i}\right)=\min \left\{f(\omega)+y_{i} \omega\left(A^{(i)}\right) \mid \omega \in \mathscr{S}(\mathscr{A}) ; n(\omega)=\bar{\varrho}_{i}\right\},
$$

i.e., by Eq. (3.4),

$$
\psi\left(\bar{\varrho}_{i}, y_{i}\right)=\min \left\{\varphi\left(\bar{\varrho}_{i}, \alpha_{i}\right)+y_{i} \alpha_{i} \mid \alpha_{i} \in \mathbf{R}\right\} .
$$

It follows from (A1.21), (A 1.22) that

$$
\psi\left(\bar{\varrho}_{i}, y_{i}\right)-\psi\left(\bar{\varrho}_{i}, \bar{y}_{i}\right) \leqq\left(y_{i}-\bar{y}_{i}\right) \bar{\alpha}_{i},
$$

and from (A1.18) and (A1.22) that $\left\{\bar{F}_{N}^{(0)}\right\}$ has an accumulation point, namely

$$
\bar{F}^{(0)}=\sum_{i=1,2}\left(\psi\left(\bar{\varrho}_{i}, \bar{y}_{i}\right)-\alpha_{i} \bar{y}_{i}\right)+\bar{\alpha}_{1} \bar{\alpha}_{2}+v \bar{\varrho}_{1} \bar{\varrho}_{2} .
$$

It now remains for us to prove that $\bar{F}^{(0)}$ is equal to the R.H.S. of (A 1.5). 
Let $\Delta_{i}$ be the convex set given by $\left\{\alpha_{i} \in R \mid \psi\left(\bar{\varrho}_{i}, y_{i}\right)-\psi\left(\bar{\varrho}_{i}, \bar{y}_{i}\right) \leqq\left(y_{i}-\bar{y}_{i}\right) \alpha_{i} \forall y_{i} \in \mathbf{R}\right\}$ corresponding to the set of tangents to $\psi\left(\bar{\varrho}_{i}, \cdot\right)$ at $\bar{y}_{i}$; and let $\mathscr{E}\left(\Delta_{i}\right)$ be the set of extremal elements of $\Delta_{i}$. Then since by (A1.24), $\bar{\alpha}_{i} \in \Delta_{i}$, we may write

$$
\bar{\alpha}_{i}=\sum_{j} c_{j} \bar{\alpha}_{i j} ; c_{j}>0 ; \sum_{j} c_{j}=1 ; \bar{\alpha}_{i j} \in \mathscr{E}\left(\Delta_{i}\right)
$$

Further, by [9; Theorem 1], as $\bar{\alpha}_{i j} \in \mathscr{E}\left(\Delta_{i}\right)$, there exist sequences $\left\{\bar{y}_{i j n}\right\},\left\{\bar{\alpha}_{i j n}\right\}$, converging to $\bar{y}_{i}, \bar{\alpha}_{i j}$, respectively, such that $\psi\left(\bar{\varrho}_{i}, y_{i}\right)$ is differentiable w.r.t. $y_{i}$ at $\bar{y}_{i j n}$ and that the resultant differential coefficient is $\bar{\alpha}_{i j n}$. On the other hand, one may infer from (A1.23) and the concavity of $\psi$ that when $y_{i}=\bar{y}_{i j n}$, the term on the R.H.S. of that equation is minimised at $\psi\left(\bar{\varrho}_{i}, \bar{y}_{i j n}\right):=\bar{\alpha}_{i j n}$, where $\psi_{y}$ denotes the derivative of $\psi$ w.r.t. its second argument. Hence, by (A1.23)

$$
\varphi\left(\bar{\varrho}_{i}, \bar{\alpha}_{i j n}\right)=\psi\left(\bar{\varrho}_{i}, \bar{y}_{i j n}\right)-\bar{y}_{i j n} \bar{\alpha}_{i j n},
$$

and therefore, by (A1.26), (A1.27)

$$
\begin{aligned}
\psi\left(\bar{\varrho}_{i}, \bar{y}_{i}\right)-\bar{y}_{i} \bar{\alpha}_{i} & =\lim _{n \rightarrow \infty} \sum_{j} c_{j} \varphi\left(\bar{\varrho}_{i}, \bar{\alpha}_{i j n}\right) \\
& \left.\geqq \limsup _{n \rightarrow \infty} \varphi\left(\bar{\varrho}_{i}, \sum_{j} c_{j} \bar{\alpha}_{i j n}\right) \quad \text { (convexity of } \varphi\right) \\
& \geqq \limsup _{n \rightarrow \infty} \hat{\varphi}\left(\bar{\varrho}_{i}, \sum_{j} c_{j} \bar{\alpha}_{i j n}\right) \quad(\text { as } \varphi \geqq \hat{\varphi}) \\
& \geqq \hat{\varphi}\left(\bar{\varrho}_{i}, \bar{\alpha}_{i}\right) \quad[\text { by (A1.26) and lower semicontinuity of } \hat{\varphi}]
\end{aligned}
$$

Hence, by (A 1.6) and (A1.25),

$$
\bar{F}^{(0)} \geqq \hat{\Phi}\left(\bar{\varrho}_{1}, \bar{\varrho}_{2} ; \bar{\alpha}_{1}, \bar{\alpha}_{2}\right) \text {. }
$$

Let $\hat{\varphi}^{\prime}: \mathbf{R}_{+} \times \mathbf{R} \rightarrow \mathbf{R} \cup\{\infty\}$ be defined so that, for fixed $\varrho, \hat{\varphi}^{\prime}(\varrho, \cdot)$ is the closure of $\varphi(\varrho, \cdot)$; and let $\Phi, \hat{\Phi}^{\prime}$ be the function obtained by replacing $\hat{\varphi}$ by $\varphi, \hat{\varphi}^{\prime}$ in the definition of $\hat{\Phi}$ in (A1.6). It follows easily from these definitions that $\Phi \geqq \hat{\Phi}^{\prime} \geqq \hat{\Phi}$ and that the minimum of $\hat{\Phi}$ may be replaced by the infinum of $\Phi$, and thus also by the infimum of $\Phi^{\prime}$, in (A1.6). Hence, in view of (A1.28), we see that the desired result will be established if we prove that, for arbitrary fixed $\varrho_{1}, \varrho_{2}>0$, with $\varrho_{1}+\varrho_{2}=1$,

$$
\min _{\alpha_{1}, \alpha_{2}} \hat{\Phi}^{\prime}\left(\varrho_{1}, \varrho_{2} ; a_{1}, \alpha_{2}\right) \geqq \bar{F}^{(0)}
$$

Let $\hat{\Phi}^{\prime}\left(\varrho_{1}, \varrho_{2} ; \cdot\right)$ attain its minimum at $\left(\hat{\alpha}_{1}, \hat{\alpha}_{2}\right)$. Then, defining

$$
\hat{y}_{1}=\hat{\alpha}_{2} ; \hat{y}_{2}=\hat{\alpha}_{1} \text {, }
$$

it follows from our definition of $\hat{\Phi}^{\prime}$ that $\hat{\varphi}^{\prime}\left(\varrho_{i}, \alpha_{i}\right)+\hat{y}_{i} \alpha_{i}$ is minimised at $\hat{\alpha}_{i}$. Thus

$$
\begin{aligned}
\hat{\varphi}^{\prime}\left(\varrho_{i}, \hat{\alpha}_{i}\right)+\hat{y}_{i} \hat{\alpha}_{i} & =\min _{\alpha_{i}}\left(\hat{\varphi}^{\prime}\left(\varrho_{i}, \alpha_{i}\right)+\hat{y}_{i} \alpha_{i}\right)=\inf _{\alpha_{i}}\left(\varphi\left(\varrho_{i}, \alpha_{i}\right)+\hat{y}_{i} \alpha_{i}\right) \\
& =\psi\left(\varrho_{i}, \hat{y}_{i}\right), \quad \text { by (A1.23). }
\end{aligned}
$$

Hence the L.H.S. of (A1.29) is equal to

$$
\sum_{i=1,2}\left(\psi\left(\varrho_{i}, \hat{y}_{i}\right)-\hat{y}_{i} \hat{\alpha}_{i}\right)+\hat{\alpha}_{1} \hat{\alpha}_{2}+v \varrho_{1} \varrho_{2}
$$


Again we use [9; Theorem 1] and approximate $\hat{y}_{i}, \hat{\alpha}_{i}$ arbitrarily closely by $\hat{y}_{i k}$, $\sum_{k} c_{i k} \hat{\alpha}_{i k}$, with $\hat{\alpha}_{i k}=\psi_{j}\left(\varrho_{i}, \hat{y}_{i k}\right), c_{i k}>0, \sum_{k} c_{i k}=1$. Thus,

$$
\psi\left(\varrho_{i}, \hat{y}_{i}\right)-\hat{y}_{i} \hat{\alpha}_{i} \geqq \sum_{k} c_{i k}\left[\psi\left(\varrho_{i}, \hat{y}_{i k}\right)-\hat{\alpha}_{i k} \hat{y}_{i k}\right]-\varepsilon,
$$

where $\varepsilon$ may be made arbitrarily small by choosing $\left\{\hat{y}_{i k}\right\}$ sufficiently close to $\hat{y}_{i}$. Further [cf. (A1.22)]

$$
\psi\left(\varrho_{i}, \hat{y}_{i k}\right)=\lim _{N \rightarrow \infty} \psi_{N}\left(\varrho_{i}^{(N)}, \hat{y}_{i k}\right) \text {; with } \lim _{N \rightarrow \infty} \varrho_{i}^{(N)}=\varrho_{i} .
$$

Hence, as $\psi\left(\varrho_{i}, \cdot\right)$ is differentiable at $\hat{y}_{i k}$ and $\psi_{N}\left(\varrho_{i}^{(N)}, \cdot\right)$ is differentiable at all points, it follows (by Lemma 3 ) that

$$
\lim _{N \rightarrow \infty} \hat{\alpha}_{i k}^{(N)}=\hat{\alpha}_{i k}
$$

with

$$
\hat{\alpha}_{i k}^{(N)}:=\psi_{N, y}\left(\varrho_{i}, \hat{y}_{i k}^{(N)}\right) .
$$

Consequently, by (A1.19) and (A1.32)-(A1.35),

$$
\psi\left(\varrho_{i}, \hat{y}_{i}\right)-\hat{y}_{i} \hat{\alpha}_{i} \geqq \lim _{N \rightarrow \infty} N^{-1} \sum_{k} c_{i k} \tilde{T}_{i}\left(\hat{\sigma}_{i k} \ln \hat{\sigma}_{i k}+\hat{\sigma}_{i k} \tilde{T}_{i}\right)-\varepsilon,
$$

where

$$
\hat{\sigma}_{i k}=\exp -\left(\tilde{T}_{i}+\hat{y}_{i k} \tilde{A}_{i}^{(i)}\right) / \tilde{T}_{i}(\text { idem }),
$$

and thus

$$
\tilde{T}_{i}\left(\hat{\sigma}_{i k} \tilde{A}_{i}^{(i)}\right)=\hat{\alpha}_{i k}^{(N)},
$$

where $\operatorname{Tr}_{i}$ is the Trace over the $N \varrho_{i}^{(N)}$ particle subspace of $\mathscr{H}\left(\tilde{C}_{i}\right)$. Putting $\hat{\sigma}_{i}$ $=\sum_{k} c_{i k} \hat{\sigma}_{i k}$, it follows from (A1.36), together with the convexity of $\tilde{T}_{i}\left(\tilde{\sigma}_{i} \ln \tilde{\sigma}_{i}\right)$ in $\tilde{\sigma}_{i}$, that

$$
\psi\left(\varrho_{i}, \hat{y}_{i}\right) \geqq \limsup _{N \rightarrow \infty} N^{-1} \tilde{\operatorname{Tr}}_{i}\left(\hat{\sigma}_{i} \ln \hat{\sigma}_{i}+\hat{\sigma}_{i} \tilde{T}_{i}\right)-\varepsilon .
$$

Thus, by (A1.1), (A1.38) and (A1.39), the expression (A1.31) is not less than

$$
\limsup _{N \rightarrow \infty} N^{-1} \tilde{\operatorname{Tr}}\left(\hat{\sigma} \ln \hat{\sigma}+\hat{\sigma} \tilde{H}_{N, \varrho}\right)-\varepsilon, \quad \text { with } \quad \hat{\sigma}=\hat{\sigma}_{1} \otimes \hat{\sigma}_{2}
$$

Consequently, by (A1.3) and our definition of $\bar{F}^{(0)}$ as a limit point of $\left\{\bar{F}_{N}^{(0)}\right\}$, the expression (A1.31) cannot be less than $\bar{F}^{(0)}-\varepsilon$; and, as $\varepsilon$ is arbitrary, this means that (A 1.29) is valid.

\section{Appendix 2}

For $\varrho \in \mathbf{R}_{+}$, we define $\Delta_{\varrho}:=\left\{\omega \in \mathscr{S}(\mathscr{A}) \mid f(\omega)=\varphi_{0}(\varrho) ; n(\omega)=\varrho\right\}$, corresponding to the set of translationally invariant equilibrium states of the ideal Fermi gas. We shall prove the following theorem. 
Theorem A2.1. $\Delta_{\varrho}$ consists of a single element, and this satisfies the K.M.S. conditions with respect to the free evolution of the ideal Fermi gas.

Our proof of this theorem will be based on constructions, analogous to those made for lattice systems in [9]. Thus, we first resolve $\mathbf{R}^{3}$ into (half-open) cubes, whose centres are the sites of the lattice $\mathbf{Z}^{3}$, and define $\mathscr{Y}$ to be the set of bounded subsets $\{Y\}$ of $\mathbf{R}^{3}$, formed by unions of finite numbers of these cubes. We then define $\mathscr{B}$ to be the set of mappings $b$ from $\mathscr{Y}$ into the self-adjoint elements of $\mathscr{A}$ such that (i) $b(Y) \in \mathscr{A}(Y) \forall Y \in \mathscr{Y}$; (ii) $b$ is covariant w.r.t. space translations, i.e. $b(Y+n)=\tau(n) b(Y) \forall Y \in \mathscr{Y}, n \in \mathbf{Z}^{3}$, where $\tau\left(\mathbf{R}^{3}\right)$ is the group of automorphisms of $\mathscr{A}$ corresponding to space translations; and (iii)

$$
|\varphi|:=\sum_{0 \in Y}\|b(Y)\|<\infty .
$$

The set $\mathscr{B}$, equipped with the norm $|\cdot|$, is thus a separable Banach space. For $Y \in \mathscr{Y}$, we define $H(Y)$ to be the operator in $\mathscr{H}(Y)$ corresponding to the Hamiltonian for an ideal Fermi gas in $Y$, with Dirichlet boundary conditions; and we denote the $N$ particle component of $H(Y)$ by $H_{N}(Y)$. We define the local perturbative Hamiltonian, $U_{b}(Y)(\in \mathscr{A}(Y))$, corresponding to the "potential" $b$, by the formula

$$
U_{b}(Y)=\sum_{Y^{\prime} \subset Y} b\left(Y^{\prime}\right)
$$

and define the free energy density functional $\mathscr{F}_{\varrho}: \mathscr{B} \rightarrow \mathbf{R}$ by the following formula, of standard type:

$$
\mathscr{F}_{\varrho}(b)=-\lim _{Y \uparrow \mathbf{R}^{3} ; N /|Y| \rightarrow \varrho}(\beta|Y|)^{-1} \ln \operatorname{Tr}_{N} \exp -\beta\left(H(Y)+U_{b}(Y)\right),
$$

where $\operatorname{Tr}_{N}$ denotes the trace over the $N$-particle subspace of $\mathscr{H}(Y)$. Let $\tilde{\mathscr{S}}(\mathscr{A})$ denote the set of $\mathbf{Z}^{3}$-invariant states on $\mathscr{A}, \tilde{f}$ the free energy density functional on $\tilde{\mathscr{S}}(\mathscr{A})$ - defined analogously with $f$-for the ideal Fermi gas; and, for $b \in B$, let $\tilde{f}_{b}$ be the "perturbed" free energy density functional on $\tilde{\mathscr{S}}(\mathscr{A})$ given by

$$
\tilde{f}_{b}(\omega)=\tilde{f}(\omega)+\sum_{0 \in Y} \frac{\omega(b(Y))}{|Y|} .
$$

It follows from arguments parallel to those of [9] that $\mathscr{F}_{\varrho}(b)$ is the minimal value of $f_{b}$, and that the (convex compact) set of states $\Delta_{\varrho, b}$ at which $f_{b}$ attains this minimum are those elements, $\omega$, of $\tilde{\mathscr{S}}(\mathscr{A})$ corresponding to tangent planes to $\mathscr{I}_{\varrho}$ at $b$, i.e. those for which

$$
\mathscr{F}_{\varrho}\left(b+b^{\prime}\right)-\mathscr{F}_{\varrho}(b) \leqq \sum_{0 \in Y} \frac{\omega\left(b^{\prime}(Y)\right)}{|Y|} \forall b^{\prime} \in \mathscr{B} .
$$

Let $\mathscr{A}_{0}$ be the subalgebra of $\mathscr{A}$ on which $\lim _{Y \uparrow R^{3}}[H(Y), \cdot]$ exists. Then the time evolution of the ideal Fermi gas corresponds to a group $\gamma(\mathbf{R})$ of automorphisms of $\mathscr{A}$, whose generator $\delta$ has $\mathscr{A}_{0}$ as a core and is given by (cf. [15]):

$$
\delta(A)=\lim _{Y \uparrow R^{3}} i[H(Y), A] \forall A \in \mathscr{A}_{0} .
$$


Correspondingly, the KMS conditions for a state $\omega$ of the ideal Fermi gas may be expressed in the following form [16]:

$$
-i \omega\left(A^{*} \delta A\right) \geqq g\left(\omega\left(A^{*} A\right), \omega\left(A A^{*}\right)\right),
$$

where

$$
g(u, v)= \begin{cases}u \ln u-u \ln v & \text { for } \quad u, v \geqq 0 ; u+v>0 \\ 0 & \text { for } \quad u=v=0 .\end{cases}
$$

Proof of Theorem $A 2.1$. Let $\mathscr{B}_{0}$ be the subset of elements of $\mathscr{B}$ at which $\mathscr{F}_{Q}$ has a unique tangent plane. Then (cf. [9]), $\mathscr{B}_{0}$ is dense in $\mathscr{B}$, and the extremal tangent planes at 0 are given by limits of those for sequences of elements $b\left(\in \mathscr{B}_{0}\right)$ that converge to 0 . Further, for $b \in \mathscr{B}_{0}$, the unique element $\bar{\omega}_{b}$ of $\tilde{\Delta}_{\varrho, b}$ is given by the formula

$$
\bar{\omega}_{b}(A)=\lim _{Y \uparrow R^{3} ; N /|N| \rightarrow \varrho} \omega_{Y, b}^{(N)}\left(\bar{A}_{Y}\right) \forall A \in \mathscr{A}_{2},
$$

where

$$
\begin{aligned}
\omega_{Y, b}^{(N)} & =\operatorname{Tr}_{N}\left((\cdot) \exp -\beta\left(H(Y)+U_{b}(Y)\right)\right) / \operatorname{Tr}_{N}\left(\exp -\beta\left(H(Y)+U_{b}(Y)\right)\right), \\
\bar{A}_{Y} & =|Y|^{-1} \sum_{l \in \bar{Y}} A_{l} ; \quad A_{l}:=\tau(l) A ;
\end{aligned}
$$

and $\tilde{Y}$ is the set of elements $l$ of $\mathbf{Z}^{3}$ such that $\tau(l) A \in \mathscr{A}(Y)$. Since $\omega_{Y, b}^{(N)}$ is a Gibbs state on $\mathscr{A}(Y)$, it satisfies the KMS condition w.r.t. the automorphisms of that algebra, for which the generator is

$$
\delta_{Y, b}:=i\left[H(Y)+U_{b}(Y), \cdot\right] .
$$

Thus

$$
-i \omega_{Y, b}^{(N)}\left(A^{*} \delta_{Y, b} A\right) \geqq g\left(\omega_{Y, b}^{(N)}\left(A^{*} A\right), \omega_{Y, b}^{(N)}\left(A A^{*}\right)\right) .
$$

It follows from (A 2.2) that, for $A \in \mathscr{A}\left(Y_{0}\right)$,

$$
\begin{aligned}
\left\|\left[U_{b}(Y), A\right]\right\| & \leqq \sum_{Y^{\prime} \cap Y_{0} \neq \emptyset}\left\|\left[b\left(Y^{\prime}\right), A\right]\right\| \quad\left(\text { as } \mathscr{A}(Y) \quad \mathscr{A}\left(Y^{\prime}\right) \quad \text { if } Y \cap Y^{\prime}=\emptyset\right) \\
& \leqq 2\left|Y_{0}\right| \sum_{0 \in Y^{\prime}}\left\|b\left(Y^{\prime}\right)\right\|\|A\| \\
& =2\left|Y_{0}\right|\|A\||b|, \quad \text { by } \quad(\mathrm{A} 2.1) ;
\end{aligned}
$$

and hence, by (A2.6) and (A2.12),

$$
\lim _{b \rightarrow 0} \lim _{Y \uparrow R^{3}} \delta_{Y, b}(A)=\delta(A) \forall A \in \mathscr{A}_{0} .
$$

Thus, as $\delta$ commutes with $\tau(l)$, it follows from Eqs. (A2.9), (A2.14) that if $\bar{\omega}$ is the $w^{*}$-limit of $\bar{\omega}_{b}$, as $b \rightarrow 0$, then

$$
\lim _{b \rightarrow 0} \lim _{Y \uparrow R^{3} ; N /|Y| \rightarrow \varrho}|Y|^{-1} \sum_{l \in \tilde{Y}} \omega_{Y, b}^{(N)}\left(A_{l}^{*} \delta_{Y, b} A_{l}\right)=\bar{\omega}\left(A^{*} \delta A\right), \forall A \in \mathscr{A}_{0} .
$$

Hence, by (A2.13) and (A2.15),

$$
-i \bar{\omega}\left(A^{*} \delta A\right) \geqq \lim _{b \rightarrow 0} \limsup _{Y \uparrow R^{3} ; N /|Y| \rightarrow \varrho}|Y|^{-1} \sum_{l \in \tilde{Y}} g\left(\omega_{Y, b}^{(N)}\left(A_{l}^{*} A_{l}\right), \omega_{Y, b}^{(N)}\left(A_{l} A_{l}^{*}\right)\right), \forall A \in \mathscr{A}_{l} .
$$


Now as $\lim _{Y \uparrow R^{3}}|\tilde{Y}| /|Y|=1$, and as the function $g$ is jointly convex in its arguments and possesses the property that, for $u_{n} \rightarrow u$ and $v_{n} \rightarrow v$, $\lim \inf g\left(u_{n}, v_{n}\right) \geqq g(u, v)$, it follows from (A2.9)-(A2.11) and (A2.16) that

$$
-i \omega\left(A^{*} \delta A\right) \geqq g\left(\bar{\omega}\left(A^{*} A\right), \omega\left(A A^{*}\right)\right) \forall A \in \mathscr{A}_{0},
$$

and therefore $\bar{\omega}$ satisfies the KMS conditions.

Let $\tilde{\mathscr{A}}(\supset \mathscr{A})$ be the gauge-dependent $C^{*}$-algebra of the CAR over $L^{2}\left(\mathbf{R}^{3}\right)$, and let $\tilde{\omega}_{\mu}$ be the unique (cf. [17] KMS state on $\tilde{\mathscr{A}}$ corresponding to chemical potential $\mu$. Then, as $\bar{\omega}$ is a KMS state on $\mathscr{A}$, it follows [18] that we may express it in the form

$$
\bar{\omega}=\int d m(\mu) \tilde{\omega}_{\mu}
$$

where $m$ is some measure over $\mathbf{R}$. Thus, as $\tilde{\omega}_{\mu}$ is $\mathbf{R}^{3}$-translationally invariant (cf. [17]), then so too is $\bar{\omega}$. Since the functionals $n$ and $f$ are affine, it follows from the definition of $\varphi_{0}(\varrho)$ as the minimal value of $f$ for translationally invariant states of particle density $\varrho$ that

$$
\varrho=\int d m(\mu) n_{0}(\mu)
$$

and

$$
\varphi_{0}(\varrho)=\int d m(\mu) \psi_{0}(\mu)
$$

where the functions $n_{0}, \psi_{0}$ represent the densities of particle number and free energy, respectively, and are given by the standard formulae

$$
n_{0}(\mu)=\frac{1}{\pi^{2}} \int d^{3 k}\left[\exp \beta\left(\frac{1}{2} k^{2}-\mu\right)+1\right]^{-1}
$$

and

$$
\psi_{0}(\mu)=\frac{1}{\pi^{2}} \int d^{3} k\left[-\beta^{-1} \ln \left(1+\exp -\beta\left(\frac{1}{2} k^{2}-\mu\right)\right)+\mu\left(1+\exp \beta\left(\frac{1}{2} k^{2}-\mu\right)\right)^{-1}\right] .
$$

From (A 1.20), one infers easily that the function $n_{0}$ is single-valued and invertible; and thus, in view of the equivalence of ensembles [10],

$$
\varphi_{0}(\varrho)=\psi_{0}\left(\mathrm{n}_{0}^{-1}(\varrho)\right) \text {. }
$$

Hence, by (A2.19),

$$
\varphi_{0}(\varrho)=\int d v\left(\varrho^{\prime}\right) \varphi_{0}\left(\varrho^{\prime}\right)
$$

where

$$
d v\left(n_{0}(\mu)\right)=d m(\mu) .
$$

Further, one can infer easily from (A2.20)-(A2.22) that $\varphi_{0}$ is strictly convex in $\varrho$; and consequently, by (A2.23), $v$ must be the Dirac measure, with support at $\varrho$. Hence, by (A2.17) and (A2.24), $\bar{\omega}=\tilde{\omega}_{n_{0}{ }^{1}(\varrho)}$. Thus, we have proved that $\tilde{\omega}_{n_{0}{ }^{1}(\varrho)}$ is the unique extremal element, and hence the unique element of $\Delta_{\varrho, 0}$. Therefore as this state is also $\mathbf{R}^{3}$-translationally invariant, it follows that it is the unique element of $\Delta_{\varrho}$. 
Acknowledgements. The authors would like to express their appreciation to B. Baumgartner, A.Pflug and $\mathrm{W}$. Thirring for a number of discussions on the subject-matter of this article, and to H. Araki for various constructive criticisms of an earlier draft of it. They would also like to thank the Österreichische Akademie der Wissenschaft and the Royal Society for financial support that made their collaboration possible.

\section{References}

1. Levy-Leblond, J.M.: J. Math. Phys. 10, 806-812 (1969)

2. Hertel, P., Thirring, W.: Commun. Math. Phys. 24, 22-36 (1971)

3. Hertel, P., Thirring, W.: Thermodynamic instability of a system with gravitating fermions. In: Quanten und Felder (ed. H.P.Durr), pp. 310-323. Braunschweig: Vieweg 1971

4. Hertel, P., Narnhofer, H., Thirring, W.: Commun. Math. Phys. 28, 159-176 (1972)

5. Baumgartner, B.: Commun. Math. Phys. 48, 207-213 (1976)

6. Dell'Antonio, G.F., Doplicher, S., Ruelle, D.: Commun. Math. Phys. 2, 223-230 (1966)

7. Sewell, G.L.: Ann. Phys. 97, 55-79 (1976); Commun. Math. Phys. 55, 63-66 (1977)

8. Bogoliubov, N.N., Jr.: Physica 32, 933-944 (1966)

9. Lanford, O., Robinson, D.W.: Commun. Math. Phys. 9, 327-338 (1968)

10. Ruelle, D.: Statistical mechanics: rigorous results. New York, Amsterdam: Benjamin 1969

11. Emch, G.G.: Algebraic methods in statistical mechanics and quantum field theory. New York, London: Wiley-Interscience 1972

12. Miracle-Sole, S., Robinson, D.W.: Commun. Math. Phys. 14, 235-270 (1969)

Robinson, D.W.: The thermodynamical pressure in quantum statistical mechanics. In: Lecture notes in physics. Berlin, Heidelberg, New York: Springer 1971

Pflug, A.: Thesis, Vienna (1978)

13. Rockafeller, R.T.: Convex analysis. Princeton: Princeton University Press 1970

14. Griffiths, R.B.: J. Math. Phys. 5, 1215-1222 (1964)

15. Brattelli, O., Robinson, D.W.: Commun. Math. Phys. 42, 253-268 (1975)

16. Sewell, G.L.: Commun. Math. Phys. 55, 53-61 (1977)

Araki, H.: Mathematical problems in theoretical physics, pp.94-105. Proceedings of the 1977 Rome Conference (eds. G.Dell'Antonio, S.Doplicher, G.Jona-Lasinio). Berlin, Heidelberg, New York: Springer 1978

Fannes, M., Verbeure, A.: Commun. Math. Phys. 57, 165-171 (1977)

17. Rocca, F., Sirugue, M., Testard, D.: Commun. Math. Phys. 13, 317-334 (1969)

18. Araki, H., Haag, R., Kastler, D., Takesaki, M.: Commun. Math. Phys. 53, 97-134 (1977)

Communicated by H. Araki

Received November 20, 1978; in revised form August 31, 1979 